\title{
The effective action of warped M-theory reductions with higher derivative terms - part I
}

\author{
Thomas W. Grimm, Tom G. Pugh and Matthias Weissenbacher \\ Max Planck Institute for Physics, \\ Föhringer Ring 6, 80805 Munich, Germany \\ E-mail: grimm@mpp.mpg.de, mweisse@mpp.mpg.de, pught@mpp.mpg.de
}

ABSTRACT: M-theory accessed via eleven-dimensional supergravity admits globally consistent warped solutions with eight-dimensional compact spaces if background fluxes and higher derivative terms are considered. The internal background is conformally Kähler with vanishing first Chern class. We perturb these solutions including a finite number of Kähler deformations of the metric and vector deformations of the M-theory three-form. Special emphasis is given to the field-dependence of the warp-factor and the higher-derivative terms. We show that the three-dimensional two-derivative effective action takes a surprisingly simple form in terms of a single higher-curvature building block due to numerous non-trivial cancellations. Both the ansatz and the effective action admit a moduli dependent scaling symmetry of the internal metric. Furthermore, we find that the required departure from Ricci-flatness and harmonicity of the zero-mode eigenforms does not alter the effective theory.

KEYWORDS: Flux compactifications, Supergravity Models, Supersymmetric Effective Theories, M-Theory

ARXIV EPRINT: 1412.5073 


\section{Contents}

1 Introduction and discussion 1

2 Eleven-dimensional action and compactifying solutions 3

2.1 The eleven-dimensional action with higher-derivative terms 3

2.2 Compactifying warped solutions with background fluxes 5

$\begin{array}{lll}3 & \text { Perturbations of the background } & 7\end{array}$

$\begin{array}{lll}3.1 & \text { Vector modes from the M-theory three-form } & 7\end{array}$

3.2 Kähler structure deformations and the warp-factor 8

4 The three-dimensional effective action $\quad 11$

$\begin{array}{ll}\text { A Conventions, definitions, and identities } & 15\end{array}$

$\begin{array}{ll}\text { B Results of the dimensional reduction } & 17\end{array}$

$\begin{array}{ll}\text { B.1 Two derivative terms } & 17\end{array}$

$\begin{array}{lll}\text { B.2 Eight derivative terms } & 17\end{array}$

\section{Introduction and discussion}

Dimensional reductions of M-theory on compact eight-dimensional manifolds result in three-dimensional effective theories with various amounts of supersymmetry. These reductions are both of conceptual as well as phenomenological interest. A phenomenological investigation might be carried out when applying the M-theory to F-theory limit in order to lift the three-dimensional theories to four space-time dimensions for a certain class of eightdimensional manifolds [1]. From a phenomenological point of view, compactifications in which the effective theory preserves only small amounts of supersymmetry are of particular interest. For example, compactifications of M-theory and F-theory preserving four supercharges allow for background fluxes that can induce a four-dimensional chiral spectrum.

A famous class of warped solutions with background fluxes was argued to exist in [2]. Global consistency, however, requires that, in a compact scenario with background fluxes, higher-derivative terms in the eleven-dimensional action must also be included. It was subsequently shown that there are indeed solutions that solve the higher-derivative field equations [3]. More precisely, one finds that the internal background is a conformally Kähler manifold with vanishing first Chern class, but a metric that is non-Ricci-flat even when allowing for a conformal rescaling including the warp factor. This deviation is due to the possible non-harmonicity of the third Chern-form in the leading order Ricci-flat metric [4]. While a complete check of supersymmetry is still missing, it was shown in [4] 
that a modification of the eleven-dimensional gravitino variations with higher curvature terms based on $[5,6]$ vanishes on the warped background solutions. It was furthermore argued, that the warped background admits a globally defined real two-form $J^{\prime}$ and complex four-form $\Omega^{\prime}$. Separating the warp-factor, the Killing spinor equations translate into first order differential constraints on these forms, with only for an exact one-form $\overline{\mathcal{W}}_{5} .{ }^{1}$

In this paper we study the three-dimensional effective action arising when perturbing the solutions considered in $[3,4]$ by a finite number of Kähler deformations of the metric and vector deformations of the M-theory three-form. More precisely, our starting point is the bosonic part of the eleven-dimensional supergravity action of [10] corrected by the terms fourth order in the Riemann curvature known since the works [11-17], and the higherderivative terms quadratic in the M-theory three-form found in [18]. Let us stress that there are important terms of the structure $(\hat{\nabla} \hat{G})^{2} \hat{R}^{3}$, where $\hat{G}$ is the M-theory four-form field-strength and $\hat{R}$ is the Riemann curvature tensor, that have not been fully determined. They were argued to be given by a number of building blocks of index contractions [19] with 4-point amplitudes only determining part of the numerical prefactors. We thus use an expression with six unknown coefficients $a_{i}$. Remarkably, four of these unknown coefficients, denoted by $a_{3}, \ldots, a_{6}$ in the conventions of (2.10), actually do not affect our computation and drop out in the dimensional reduction. For the remaining two coefficients we suggest in section 4 the identification $a_{1}=a_{2}$, leaving only one unknown constant. This last constant $a_{1}$ might then be fixed by supersymmetry [20], and we find here that $a_{1}=7$ gives the very elegant result (4.21) for the reduced action that is used in the supersymmetry analysis of [20]. Clearly, the complete form of the $(\hat{\nabla} \hat{G})^{2} \hat{R}^{3}$ terms could also be determined by considering amplitudes with 5 and more external legs.

Given the eleven-dimensional action with higher-derivative terms we systematically construct the perturbed background order by order in a scale parameter $\alpha \propto \ell_{M}^{3}$, where $\ell_{M}$ is the eleven-dimensional Planck length. At zeroth order in $\alpha$ the background is simply a direct product of a Calabi-Yau fourfold without background fluxes and preserves four supercharges. At higher order in $\alpha$ the fluxes and higher curvature terms need to be included. The metric ansatz is modified and accordingly the mode expansion for Kähler structure perturbations of the metric and vector perturbations of the M-theory three-form is described in terms of forms non-harmonic in the zeroth order Calabi-Yau metric. We carefully keep track of all such modifications, but show that most of these modifications eventually cancel in the final three-dimensional effective action. In fact, inserting the ansatz into the higher-derivative action, we find that the kinetic terms for the deformations and vectors in the three-dimensional effective theory can be expressed using a single highercurvature building block $Z_{m \bar{m} n \bar{n}}=\frac{1}{4 !}\left(\epsilon_{8} \epsilon_{8} R^{(0) 3}\right)_{m \bar{m} n \bar{n}}$, where $R^{(0)}$ is the internal Riemann tensor in the zeroth order Calabi-Yau metric, see (3.13) for the precise form of $Z$. Let us note that $Z_{m \bar{m} n \bar{n}}$ has the same symmetries as the Riemann tensor. It contracts with $R^{\bar{m} m \bar{n} n}$ to the Hodge-dual of the fourth Chern-form, and contracting any of the index pairs with

\footnotetext{
${ }^{1}$ At the two-derivative level eleven-dimensional supergravity on SU(4) structure manifolds has recently been studied in [7].The fact that the metric is no longer Ricci flat when higher derivative couplings and $\alpha^{\prime}$-corrections are taken into account is a classical result for Calabi-Yau manifolds without background fluxes in string theory [8] and has been recently investigated for $\operatorname{Spin}(7)$ and $G_{2}$ compactifications [9].
} 
the metric one finds expressions in terms of the third Chern-form. The equivalent quantity on a Calabi-Yau threefold was found to be important in [21]. It would be interesting to examine if $Z_{m \bar{m} n \bar{n}}$ plays a special role in describing the topology of the compact eightfold.

In addition to the complications arising from reducing higher-derivative terms in the action, a proper treatment of the warp-factor turns out to be crucial. Warped compactifications of M-theory and Type IIB have been considered previously in [22-31], and were argued to be crucial in a complete understanding of the M-theory to F-theory limit for minimally supersymmetric setups [32]. In this work we perform the crucial generalization to include the higher-derivative terms, since warped compactifications with fluxes are inconsistent without these contributions. It turns out, that in this general case the modifications of the warp-factor to the lower-dimensional effective theory are significantly more involved then the ones discussed previously in the literature. Nevertheless we will be able to show that the effective theory permits a non-trivial scaling symmetry induced by rescaling the warp-factor by a field-dependent function. In a subsequent paper [20] we will argue that the three-dimensional action carries the properties of a $\mathcal{N}=2$ supergravity theory and extend the results of [33-35].

The paper is organized as follows. In section 2 we review the eleven-dimensional effective action of M-theory including higher-derivative terms. We then introduce the considered warped solutions that admit an eight-dimensional compact internal manifold and background fluxes and comment on the supersymmetry conditions. The considered perturbations of the background solutions are introduced in section 3 and consist of vector modes of the M-theory three-form and Kähler structure deformations. We also discuss the fielddependence of the warp-factor. The dimensional reduction yielding a three-dimensional effective action is carried out in section 4, where we present the results for the kinetic terms and Chern-Simons terms. A summary of our conventions and a number of useful identities are supplemented in appendix A. More details on the dimensional reduction of the higher derivative terms can be found in appendix B.

\section{Eleven-dimensional action and compactifying solutions}

In this section we introduce the eleven-dimensional action including the known higherderivative terms that will then be used in the dimensional reduction. The individual terms are discussed in subsection 2.1, with details and conventions supplemented in appendix A. The eleven-dimensional theory admits a warped solution with a compact eight-dimensional space and background fluxes as we recall in subsection 2.2.

\subsection{The eleven-dimensional action with higher-derivative terms}

Our starting point will be the eleven-dimensional supergravity action that arises as the low energy limit of M-theory. At the two-derivative level the action is the long-known $\mathcal{N}=1$ supergravity action first worked out in [10]. Recall that the dynamical fields of this supergravity theory arrange in an $\mathcal{N}=1$ gravity multiplet, with bosonic fields being the eleven-dimensional metric $\hat{g}_{N M}$ and a three-form $\hat{C}_{M N P}$ with field strength $\hat{G}_{Q M N P}=$ 
$\partial_{[Q} \hat{C}_{M N P]}$. In the following we will indicate eleven-dimensional quantities with a hat. The action for these bosonic fields is given by

$$
S^{(0)}=\frac{1}{2 \kappa_{11}^{2}} \int\left[\hat{R} \hat{*} 1-\frac{1}{2} \hat{G} \wedge \hat{*} \hat{G}-\frac{1}{6} \hat{C} \wedge \hat{G} \wedge \hat{G}\right],
$$

where $\hat{R}$ is the Ricci scalar evaluated with conventions introduced in appendix A.

In order to find globally consistent solutions with internal background fluxes for $\hat{G}$ one has to include higher-derivative corrections to the theory as we recall below. Terms that are up to eighth order in derivatives and are quadratic in $\hat{G}$ will be crucial in this discussion. To systematically display the results we introduce the dimensionful parameter

$$
\alpha^{2}=\frac{\left(4 \pi \kappa_{11}^{2}\right)^{\frac{2}{3}}}{(2 \pi)^{4} 3^{2} 2^{13}}
$$

These bosonic terms have been worked out in [11-18], such that the action takes the form

$$
S=S^{(0)}+\alpha^{2} S_{\hat{R}^{4}}^{(2)}+\alpha^{2} S_{\hat{G}^{2} \hat{R}^{3}}^{(2)}+\alpha^{2} S_{(\hat{\nabla} \hat{G})^{2} \hat{R}^{2}}^{(2)}+\mathcal{O}\left(\hat{G}^{3} \alpha^{2}\right)+\mathcal{O}\left(\alpha^{3}\right),
$$

with eight-derivative terms given by

$$
\begin{aligned}
S_{\hat{R}^{4}}^{(2)} & =\frac{1}{2 \kappa_{11}^{2}} \int\left[\left(\hat{t}_{8} \hat{t}_{8}-\frac{1}{24} \hat{\epsilon}_{11} \hat{\epsilon}_{11}\right) \hat{R}^{4} \hat{*} 1-3^{2} 2^{13} \hat{C} \wedge \hat{X}_{8}\right], \\
S_{\hat{G}^{2} \hat{R}^{3}}^{(2)} & =\frac{1}{2 \kappa_{11}^{2}} \int\left[-\left(\hat{t}_{8} \hat{t}_{8}+\frac{1}{96} \hat{\epsilon}_{11} \hat{\epsilon}_{11}\right) \hat{G}^{2} \hat{R}^{3} \hat{*} 1\right], \\
S_{(\hat{\nabla} \hat{G})^{2} \hat{R}^{2}}^{(2)} & =\frac{1}{2 \kappa_{11}^{2}} \int \hat{s}_{18}(\hat{\nabla} \hat{G})^{2} \hat{R}^{2} \hat{*} 1 .
\end{aligned}
$$

The terms at higher order in $\hat{G}$ and $\alpha$ will not be needed in what follows as their contribution is higher order in $\alpha$ when evaluated on the ansatz we will make.

Let us now discuss the various couplings in (2.4)-(2.6) in more detail. In (2.4) we make the definitions

$$
\hat{X}_{8}=\frac{1}{192}\left(\operatorname{Tr} \hat{\mathcal{R}}^{4}-\frac{1}{4}\left(\operatorname{Tr} \hat{\mathcal{R}}^{2}\right)^{2}\right),
$$

where $\hat{\mathcal{R}}$ is the eleven-dimensional curvature two-from $\hat{\mathcal{R}}^{M}{ }_{N}=\frac{1}{2} \hat{R}^{M}{ }_{N P Q} d x^{P} \wedge d x^{Q}$, and

$$
\begin{aligned}
\hat{\epsilon}_{11} \hat{\epsilon}_{11} \hat{R}^{4} & =\epsilon^{R_{1} R_{2} R_{3} M_{1} \ldots M_{8}} \epsilon_{R_{1} R_{2} R_{3} N_{1} \ldots N_{8}} \hat{R}^{N_{1} N_{2}}{ }_{M_{1} M_{2}} \hat{R}^{N_{3} N_{4}}{ }_{M_{3} M_{4}} \hat{R}^{N_{5} N_{6}}{ }_{M_{5} M_{6}} \hat{R}^{N_{7} N_{8}}{ }_{M_{7} M_{8}}, \\
\hat{t}_{8} \hat{t}_{8} \hat{R}^{4} & =\hat{t}_{8}^{M_{1} \ldots M_{8}} \hat{t}_{8 N_{1} \ldots N_{8}} \hat{R}^{N_{1} N_{2}}{ }_{M_{1} M_{2}} \hat{R}^{N_{3} N_{4}}{ }_{M_{3} M_{4}} \hat{R}^{N_{5} N_{6}}{ }_{M_{5} M_{6}} \hat{R}^{N_{7} N_{8}}{ }_{M_{7} M_{8}},
\end{aligned}
$$

where $\epsilon_{11}$ is the eleven-dimensional totally anti-symmetric epsilon tensor and $t_{8}$ is given explicitly in (A.3) in appendix A. Using $\epsilon_{11}$ and $t_{8}$ the explicit form for the terms in (2.5) is given by

$$
\begin{aligned}
& \hat{\epsilon}_{11} \hat{\epsilon}_{11} \hat{G}^{2} \hat{R}^{3}=\hat{\epsilon}^{R M_{1} \ldots M_{10}} \hat{\epsilon}_{R N_{1} \ldots N_{10}} \hat{G}^{N_{1} N_{2}} M_{1} M_{2} \hat{G}^{N_{3} N_{4}}{ }_{M_{3} M_{4}} \hat{R}^{N_{5} N_{6}}{ }_{M_{5} M_{6}} \hat{R}^{N_{7} N_{8}}{ }_{M_{7} M_{8}} \hat{R}^{N_{9} N_{10}}{ }_{M_{9} M_{10}} \text {, } \\
& \hat{t}_{8} \hat{t}_{8} \hat{G}^{2} \hat{R}^{3}=\hat{t}_{8}^{M_{1} \ldots M_{8}} \hat{t}_{8 N_{1} \ldots N_{8}} \hat{G}^{N_{1}}{ }_{M_{1} R_{1} R_{2}} \hat{G}^{N_{2}}{ }_{M_{2}}{ }^{R_{1} R_{2}} \hat{R}^{N_{3} N_{4}}{ }_{M_{3} M_{4}} \hat{R}^{N_{5} N_{6}}{ }_{M_{5} M_{6}} \hat{R}^{N_{7} N_{8}}{ }_{M_{7} M_{8}} \text {. }
\end{aligned}
$$


Finally, we need to introduce the tensor $\hat{s}_{18}^{N_{1} \ldots N_{18}}$ appearing in (2.6). Unfortunately, the precise form of $\hat{s}_{18}$ is not known. However, one can fix significant parts of it following [19]. In order to express these parts we use the basis $B_{i}, i=1, \ldots, 24$ of [19], that labels all unrelated index contractions in $\hat{s}_{18}(\hat{\nabla} \hat{G})^{2} \hat{R}^{2}$. The $B_{i}$ are explicitly given in (A.4). The result can then be expressed in terms of a 4-point amplitude contribution $\mathcal{A}$ and a linear combination of six contributions $\mathcal{Z}_{i}$ which do not affect the 4-point amplitude as

$$
\hat{s}_{18}(\hat{\nabla} \hat{G})^{2} \hat{R}^{2}=\hat{s}_{18}^{N_{1} \ldots N_{18}} \hat{R}_{N_{1} \ldots N_{4}} \hat{R}_{N_{5} \ldots N_{8}} \hat{\nabla}_{N_{9}} \hat{G}_{N_{10} \ldots N_{13}} \hat{\nabla}_{N_{14}} \hat{G}_{N_{15} \ldots N_{18}}=\mathcal{A}+\sum_{n} a_{n} \mathcal{Z}_{n} .
$$

The combinations $\mathcal{A}$ and $\mathcal{Z}_{n}$ are then given in terms of the basis elements as

$$
\begin{aligned}
\mathcal{A}= & -24 B_{5}-48 B_{8}-24 B_{10}-6 B_{12}-12 B_{13}+12 B_{14}+8 B_{16}-4 B_{20}+B_{22}+4 B_{23}+B_{24}, \\
\mathcal{Z}_{1}= & 48 B_{1}+48 B_{2}-48 B_{3}+36 B_{4}+96 B_{6}+48 B_{7}-48 B_{8}+96 B_{10} \\
& \quad+12 B_{12}+24 B_{13}-12 B_{14}+8 B_{15}+8 B_{16}-16 B_{17}+6 B_{19}+2 B_{22}+B_{24}, \\
\mathcal{Z}_{2}= & -48 B_{1}-48 B_{2}-24 B_{4}-24 B_{5}+48 B_{6}-48 B_{8}-24 B_{9}-72 B_{10}-24 B_{13}+24 B_{14}-B_{22}+4 B_{23}, \\
\mathcal{Z}_{3}= & 12 B_{1}+12 B_{2}-24 B_{3}+9 B_{4}+48 B_{6}+24 B_{7}-24 B_{8}+24 B_{10} \\
& \quad+6 B_{12}+6 B_{13}+4 B_{15}-4 B_{17}+3 B_{19}+2 B_{21}, \\
\mathcal{Z}_{4}= & 12 B_{1}+12 B_{2}-12 B_{3}+9 B_{4}+24 B_{6}+12 B_{7}-12 B_{8}+24 B_{10}+3 B_{12}+6 B_{13}+4 B_{15}-4 B_{17}+2 B_{20}, \\
\mathcal{Z}_{5}= & 4 B_{3}-8 B_{6}-4 B_{7}+4 B_{8}-B_{12}-2 B_{14}+4 B_{18}, \\
\mathcal{Z}_{6}= & B_{4}+2 B_{11} .
\end{aligned}
$$

We will show in this work that the terms $\mathcal{Z}_{3}$ to $\mathcal{Z}_{6}$ vanish both on the considered background solution and their perturbed cousins to the order in $\alpha$ we are considering. This implies that the coefficients $a_{3}-a_{6}$ are irrelevant for our analysis. For the remaining $a_{1}, a_{2}$ are suggested to be fixed to $a_{1}=a_{2}=7$ as explained in detail in section 4 . In the next subsection we discuss the solutions in more detail.

\subsection{Compactifying warped solutions with background fluxes}

In the following we will review the warped solutions following [3, 4]. The starting point are the field equations derived from the action (2.3). These have a solution with an elevendimensional metric background

$$
d \hat{s}^{2}=e^{\alpha^{2} \Phi^{(2)}}\left(e^{-2 \alpha^{2} W^{(2)}} \eta_{\mu \nu} d x^{\mu} d x^{\nu}+2 e^{\alpha^{2} W^{(2)}} g_{m \bar{n}} d y^{m} d y^{\bar{n}}\right)+\mathcal{O}\left(\alpha^{3}\right),
$$

where $\eta_{\mu \nu}$ is the three-dimensional Minkowski metric and

$$
g_{m \bar{n}}=g_{m \bar{n}}^{(0)}+\alpha^{2} g_{m \bar{n}}^{(2)}+\mathcal{O}\left(\alpha^{3}\right) .
$$

In the following we will denote the internal compact manifold by $Y_{4}$. Here $\Phi^{(2)}$ and $W^{(2)}$ are scalar function on the internal space. $\Phi^{(2)}$ represents an eleven-dimensional Weyl rescaling that will be given in terms of the internal space Riemann tensor below. $W^{(2)}$ is known as the warp-factor and generally cannot be given explicitly, but rather is constraint by a differential equation (2.20) known as the warp-factor equation. In order to give the expansion (2.13) we note that at zeroth order in $\alpha$ the background is a direct product and $g_{m \bar{n}}^{(0)}$ is a Ricci flat metric. In fact, supersymmetry of the background at lowest order in $\alpha$ demands 
that the metric $g_{m \bar{n}}^{(0)}$ must be that of a Calabi-Yau fourfold. We therefore can introduce complex indices, which here and in the following always refer to the zeroth order complex structure on the internal manifold. On a Calabi-Yau fourfold there exists a nowhere vanishing covariantly constant Kähler form $J^{(0)}$ and holomorphic $(4,0)$-form $\Omega^{(0)}$ satisfying

$$
d J^{(0)}=d \Omega^{(0)}=0 .
$$

In what follows we will work in conventions in which the internal space indices are raised and lowered with the lowest order internal space metric $g_{m \bar{n}}^{(0)}$.

The background also includes a flux for the four-form given by

$$
\begin{array}{ll}
\hat{G}_{m \bar{n} r \bar{s}}=\alpha G_{m \bar{n} r \bar{s}}^{(1)}+\mathcal{O}\left(\alpha^{3}\right), & \hat{G}_{m n r s}=\alpha G_{m n r s}^{(1)}+\mathcal{O}\left(\alpha^{3}\right), \\
\hat{G}_{\mu \nu \rho m}=\epsilon_{\mu \nu \rho} \partial_{m} e^{-3 \alpha^{2} W^{(2)}}+\mathcal{O}\left(\alpha^{3}\right) . &
\end{array}
$$

In order that the eleven-dimensional field equations are solved to order $\alpha^{2}$ by this background the flux $G^{(1)}$ must be self-dual in the lowest-order metric $g_{m \bar{n}}^{(0)}$. This condition allows $(2,2)$ and $(4,0)+(0,4)$ components of the flux with respect to the lowest order complex structure.

The analysis of the higher derivative equations of motion fixes the value of the elevendimensional Weyl rescaling $\Phi^{(2)}$ in terms of the lowest order metric $g_{m \bar{n}}^{(0)}$ as

$$
\Phi^{(2)}=-\frac{512}{3} Z, \quad Z=*^{(0)}\left(J^{(0)} \wedge c_{3}^{(0)}\right),
$$

where $c_{3}^{(0)}$ is the third Chern form built from $g_{m \bar{n}}^{(0)}$. As $c_{3}^{(0)}$ is a closed real six-form on a Kähler manifold we may write

$$
c_{3}^{(0)}=H^{(0)} c_{3}^{(0)}+i \partial^{(0)} \bar{\partial}^{(0)} F,
$$

where $H^{(0)}$ indicates the projection to the harmonic part associated with the metric $g_{m \bar{n}}^{(0)}$. Using this decomposition we note that the scalar $Z$ is given by

$$
Z=*^{(0)}\left(J^{(0)} \wedge H^{(0)} c_{3}^{(0)}\right)+\frac{1}{4} \Delta^{(0)} *^{(0)}\left(J^{(0)} \wedge J^{(0)} \wedge F\right) .
$$

The higher-derivative Einstein equations then fix the metric correction to be

$$
g_{m \bar{n}}^{(2)}=768 \partial_{m}^{(0)} \bar{\partial}_{\bar{n}}^{(0)} \tilde{F}, \quad \quad \tilde{F}=*^{(0)}\left(J^{(0)} \wedge J^{(0)} \wedge F\right) .
$$

This implies that the metric $g_{m \bar{n}}$ introduced in (2.13) is still Kähler and that the internal part of the eleven-dimensional metric (2.12) is conformally Kähler. The field equations for the M-theory three-form $\hat{C}$ and the external space Einstein equations then constrain the warp-factor $W^{(2)}$ to satisfy

$$
d^{\dagger} d e^{3 \alpha^{2} W^{(2)}}+\alpha^{2} \frac{1}{2} G^{(1)} \wedge G^{(1)}+3^{2} 2^{13} \alpha^{2} X_{8}+\mathcal{O}\left(\alpha^{3}\right)=0
$$

With these expressions one can demonstrate that all eleven-dimensional equations of motion are indeed satisfied [3, 4]. For a compact $Y_{4}$ the warp-factor equation $(2.20)$ implies the global consistency condition

$$
\frac{1}{3^{2} 2^{14}} \int_{Y_{4}} G^{(1)} \wedge G^{(1)}=\frac{\chi\left(Y_{4}\right)}{24}
$$


where $\chi\left(Y_{4}\right)=-4 ! \int_{Y_{4}} X_{8}$ is the Euler number of $Y_{4}$. Using self-duality of the fluxes $G^{(1)}$ one thus realizes that in higher-derivative terms cannot be consistently ignored if one allows for a background flux. The somewhat unusual numerical factor in (2.21) stems from our normalization of $G^{(1)}$ with $\alpha$ and can be removed when moving to quantized fluxes $G^{\text {flux }}=\frac{1}{32^{6} \sqrt{2}} G^{(1)}$.

Let us close this section with a short discussion on supersymmetry. It should be stressed that the full supersymmetric completion of the action (2.3) is not known and neither have the supersymmetry variations of the fermions been written down. In [4] a proposal was made for the gravitino variations including order $\alpha^{2}$-terms based on $[5,6]$. It was shown to be compatible with the Einstein equations. At linear order in $\alpha$ the supersymmetry variations were unchanged and the condition on the flux is the vanishing of the $(4,0)+(0,4)$-component of $G^{(1)}$, i.e.

$$
G_{m n r s}^{(1)}=0
$$

and the primitivity condition

$$
G^{(1)} \wedge J^{(0)}=0 .
$$

It was also argued in [4] that the presented solution for the metric is compatible with the proposed Killing spinor equations at order $\alpha^{2}$. Since we will not bring the three-dimensional effective action into standard $\mathcal{N}=2$ form, the discussion of supersymmetry will not be crucial in this work.

\section{Perturbations of the background}

In subsection 2.2 we have reviewed a supersymmetric background with an internal compact space that is conformally Kähler. We will now examine a set of deformations that preserve the Kähler condition but change the chosen Kähler structure. Our whole discussion will be carried out at fixed complex structure, i.e. there are no complex structure deformations that will be switched on. In the following, the complex structure is chosen such that the supersymmetry condition (2.22) on the flux is satisfied. At lowest order in $\alpha$ the Kähler structure deformations are known to combine with vectors arising from the Mtheory three-form $\hat{C}$ into three-dimensional $\mathcal{N}=2$ multiplets, as discussed e.g. in [36, 37]. We therefore need to study vectors arising from $\hat{C}$ taking into account higher $\alpha$-corrections in subsection 3.1. The real scalars $v^{i}$ that correspond to the deformations of the Kähler structure will be introduced in subsection 3.2. In this latter subsection we will also study the variations of the warp-factor equation with respect to the Kähler structure deformations.

\subsection{Vector modes from the M-theory three-form}

Let us first examine the vector which arises in perturbations of the M-theory three-form $\hat{C}$. These correspond to a extra terms in the expansion of $\hat{G}$ of the form

$$
\delta \hat{G}=F^{i} \wedge \omega_{i}^{(\mathrm{v})},
$$


where $F^{i}=d A^{i}$ and so provides the field strength for a three-dimensional vector $A^{i}$, and $\omega_{i}^{(\mathrm{v})}$ are two-forms on the internal manifold. The tensor gauge symmetry of $\hat{G}$ translates to the $\mathrm{U}(1)$ gauge symmetry of the $A^{i}$ in the three-dimensional effective theory.

In order to make the meaning of (3.1) precise, we need to specify the two-forms $\omega_{i}^{(\mathrm{v})}$. Therefore, as with the background fields studied in subsection 2.2, we consider the expansion of $\omega_{i}^{(\mathrm{v})}$ to order $\alpha^{2}$ as

$$
\omega_{i}^{(\mathrm{v})}=\omega_{i}^{(0)(\mathrm{v})}+\alpha^{2} \omega_{i}^{(2)(\mathrm{v})} .
$$

By making use of the Bianchi identity $d \hat{G}=0$ in the absence of localized sources we see that $d \omega_{i}^{(0)(v)}=d \omega_{i}^{(2)(v)}=0$. The standard analysis of the lowest order reduction shows that only the harmonic part of $\omega_{i}^{(0)(v)}$ contributes in the effective action and therefore we may pick $\omega_{i}^{(0)(v)}$ to be harmonic. On a Calabi-Yau fourfold this implies that $\omega_{i}^{(0)(v)}$ is a $(1,1)$ form and one has $i=1, \ldots, \operatorname{dim}\left(H^{1,1}\left(Y_{4}\right)\right)$, where $H^{1,1}\left(Y_{4}\right)$ is the $(1,1)$-form cohomology of $Y_{4}$ whose dimension is independent of the metric chosen on $Y_{4}$.

Let us next turn to $\omega_{i}^{(2)(v)}$. We first note that $\omega_{i}^{(0)(v)}$ can be redefined to absorb the harmonic part of $\omega_{i}^{(2)(v)}$. This implies that $\omega_{i}^{(2)(v)}$ must be exact and as it is a real two-form on a Kähler manifold the $\partial \bar{\partial}$-lemma implies that it can be obtained by a $\partial^{(0)} \bar{\partial}^{(0)}$ of a scalar $\rho_{i}^{(\mathrm{v})}$. In other words, one can write

$$
\omega_{i}^{(0)(\mathrm{v})}=H^{(0)} \omega_{i}^{(0)(\mathrm{v})}, \quad \quad \omega_{i}^{(2)(\mathrm{v})}=\partial^{(0)} \bar{\partial}^{(0)} \rho_{i}^{(\mathrm{v})} .
$$

The scalars $\rho_{i}^{(\mathrm{v})}$ parametrizes our ignorance in incorporating the higher-derivative corrections in the ansatz for the three-dimensional vector perturbations. Strictly speaking the indices $i$ on the $\rho_{i}^{(\mathrm{v})}$ and hence $\omega_{i}^{(2)(\mathrm{v})}$ and $\omega_{i}^{(\mathrm{v})}$ are not restricted to the range $1, \ldots, \operatorname{dim}\left(H^{1,1}\left(Y_{4}\right)\right)$ as before. However, as we will see in the explicit derivation of the effective action, all $\rho_{i}^{(\mathrm{v})}$ actually drop out of the final expression and therefore cannot yield additional dynamical fields. Interestingly, there is also a particular choice $\rho_{i}^{(\mathrm{v})}$ one could imagine, where $\omega_{i}^{(\mathrm{v})}$ is harmonic with respect to the full internal space metric $(2.12)$.

\subsection{Kähler structure deformations and the warp-factor}

We now turn to the study of Kähler structure deformations of the conformally Kähler metric in (2.12). In order to do that, we introduce variations

$$
\delta g_{m \bar{n}}=i \delta v^{i} \omega_{i m \bar{n}}^{(\mathrm{s})},
$$

where $g_{m \bar{n}}$ is the Kähler metric given in (2.13). The $\delta v^{i}$ correspond to scalars in the threedimensional effective theory, while the $\omega_{i m \bar{n}}^{(s)}$ is a set of two-forms on $Y_{4}$. Despite the misuse of notation, the field-range of the index $i$ is not yet restricted. The key point is to consider only $\omega_{i m \bar{n}}^{(\mathrm{s})}$ that preserve the Kähler condition. As before we can expand the forms $\omega_{i}^{(\mathrm{s})}$ in $\alpha$ as

$$
\omega_{i}^{(\mathrm{s})}=\omega_{i}^{(0)(\mathrm{s})}+\alpha^{2} \omega_{i}^{(2)(\mathrm{s})} .
$$

Preserving the Kähler condition requires that we impose $d \omega_{i}^{(0)}=d \omega_{i}^{(2)}=0$. As before, we recall that at zeroth order in the parameter $\alpha$ the fluctuations $\delta v^{i}$ are the well-known Kähler structure deformations of the Calabi-Yau metric $g_{m \bar{n}}^{(0)}$ and the $\omega_{i}^{(0)(s)}$ can be chosen to 
be harmonic $(1,1)$-forms with $i=1, \ldots, \operatorname{dim}\left(H^{1,1}\left(Y_{4}\right)\right)$. We may then make a redefinition to absorb the harmonic part of $\omega_{i}^{(2)(\mathrm{s})}$ so that $\omega_{i}^{(2)(\mathrm{s})}=\partial^{(0)} \bar{\partial}^{(0)} \rho_{i}^{(\mathrm{s})}$. We may then redefine the $\delta v^{i}$ such that the lowest order harmonic $(1,1)$-forms match those used in the vector case

$$
\omega_{i}^{(0)(\mathrm{s})}=\omega_{i}^{(0)(\mathrm{v})}=\omega_{i}^{(0)} .
$$

Importantly the range of the index on the $\rho_{i}^{(\mathrm{s})}$ is once again a priori not restricted and there could be many more $\delta v^{i}$ than harmonic forms. However, we will again see that all the $\rho_{i}^{(\mathrm{s})}$ as well as $\tilde{F}$ appearing in (2.19) do not appear in the three-dimensional effective action. This implies that one can equally consider deformations of the form

$$
\delta g_{m \bar{n}}^{(0)}=i \delta v^{i} \omega_{i m \bar{n}}^{(0)}
$$

while making sure that all other quantities in the ansatz that are built from $g_{m \bar{n}}^{(0)}$ shift accordingly. It will be also convenient to define scalars $v^{i}$ containing the background value of $g_{m \bar{n}}^{(0)}$ by setting

$$
g_{m \bar{n}}^{(0)}+\delta g_{m \bar{n}}^{(0)}=i v^{i} \omega_{i m \bar{n}}^{(0)}
$$

There are two main complications that arise when discussing the Kähler structure deformations in a warped flux compactification. Firstly, they will in general not all be massless. Secondly, a change of Kähler structure will induce a shift in the warp-factor. The first of these points is seen at linear order in $\alpha$. When the shift (3.7) is made we see that the primitivity condition $G^{(1)} \wedge J^{(0)}=0$ given in (2.23) is not preserved by the full set of fluctuations. This means that for constant $\delta v^{i}$ the field equations do not remain solved and so the full range of $\delta v^{i}$ no longer represent massless moduli of the background. Instead the set of massless $\delta v^{i}$ now becomes those that satisfy

$$
\delta v^{i} \omega_{i}^{(0)} \wedge G^{(1)}=0 .
$$

These terms are responsible for the well known potential terms studied in the Calabi-Yau fourfold reductions with fluxes in $[36,37]$. That this result for the potential is not effected by the higher-order corrections that result from higher-curvature terms is due to the fact that the supersymmetry conditions receive no linear modification in $\alpha$ and the potential is the square of this supersymmetry constraint.

Let us now focus on the warp-factor. Going to second order in $\alpha$ we find that in addition to (2.15) the fluctuations $\delta v^{i}$ must also preserve the warp factor equation (2.20). In order that this equation is preserved by the fluctuations we must now take the warpfactor to depend both on the internal space position and also the fields $\delta v^{i}$ such that $W^{(2)}=$ $W^{(2)}\left(y^{m}, v^{i}\right)$. When we perturb the background we will then find that the derivatives of $W^{(2)}$ with respect to $v^{i}$, denoted by $\partial_{i} W^{(2)}$, appear in these equations. We will only deduce the effective action for the fluctuations $\delta v^{i}$ up to second order in $\delta v^{i}$ and therefore it will suffice to consider $W^{(2)}$ to be described by the truncated Taylor series

$$
W^{(2)}\left(y^{m}, v^{i}\right)=W^{(2)}\left|+\partial_{i} W^{(2)}\right| \delta v^{i}+\frac{1}{2} \partial_{i} \partial_{j} W^{(2)} \mid \delta v^{i} \delta v^{j},
$$


where $W^{(2)} \mid$ indicates the restriction of $W^{(2)}$ to the point in moduli space where $\delta v^{i}=0$. Demanding that (2.20) is invariant up to second order in $\delta v^{i}$ we find that at first order in $\delta v^{i}$ one has to impose

$$
\nabla^{(0) m} \nabla^{(0) \bar{n}}\left(g_{m \bar{n}}^{(0)} \partial_{i} W^{(2)}\left|-i \omega_{i m \bar{n}}^{(0)} W^{(2)}\right|+i \omega_{i}^{(0) r} r g_{m \bar{n}} W^{(2)} \mid-i 2048 \omega_{i}^{(0) \bar{s} r} Z_{m \bar{n} r \bar{s}}\right)=0,
$$

while at second order one constrains

$$
\begin{aligned}
& \nabla^{(0) m} \nabla^{(0) \bar{n}}\left(g_{m \bar{n}}^{(0)} \partial_{i} \partial_{j} W^{(2)}\left|-2 i \omega_{(i \mid m \bar{n}}^{(0)} \partial_{\mid j)} W^{(2)}\right|-2 \omega_{(i \mid m \bar{s}}^{(0)} \omega_{\mid j) \bar{s}}^{\bar{s}} W^{(2)}\left|+\omega_{i}^{(0) r}{ }_{r} \omega_{j}^{(0) s}{ }_{s} g_{m \bar{n}}^{(0)} W^{(2)}\right|\right. \\
& \left.+\omega_{i}^{(0) r}{ }_{s} \omega_{j}^{(0) s}{ }_{r} g_{m \bar{n}}^{(0)} W^{(2)} \mid-4096 \omega_{i}^{(0) \bar{s} r} \omega_{i}^{(0) \bar{t}}{ }_{\bar{t}} Z_{m \bar{n} r \bar{s}}-2048 \omega_{i}^{(0) \bar{s} t} \omega_{i t}^{(0) r} Z_{m \bar{n} r \bar{s}}+6114 Y_{i j m \bar{n}}\right)=0 .
\end{aligned}
$$

In these variational constraints we have defined

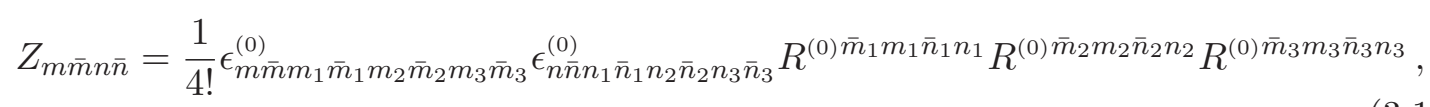

and

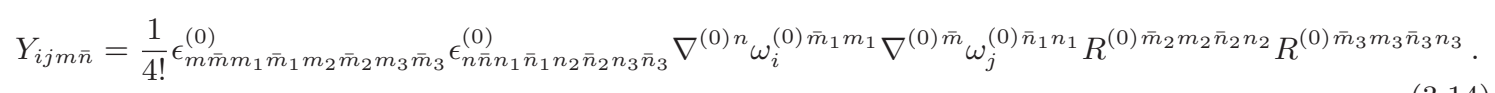

The observation that both equations (3.11) and (3.12) can be represented as total derivatives in the internal space reflects the topological nature of the terms appearing in (2.20).

It turns out that the tensor $Z_{m \bar{m} n \bar{n}}$ given in (3.13) plays a central role in the following and is related to the key topological quantities on $Y_{4}$. It satisfies the identities

$$
Z_{m \bar{m} n \bar{n}}=Z_{n \bar{m} m \bar{n}}=Z_{m \bar{n} n \bar{m}}, \quad \nabla^{(0) m} Z_{m \bar{m} n \bar{n}}=\nabla^{(0) \bar{m}} Z_{m \bar{m} n \bar{n}}=0 .
$$

It is related to the third Chern-form $c_{3}^{(0)}$ via

$$
\begin{aligned}
Z_{m \bar{m}} & =i 2 Z_{m \bar{m} n}{ }^{n}=\frac{1}{2}\left(*^{(0)} c_{3}^{(0)}\right)_{m \bar{m}}, \\
Z & =i 2 Z_{m}^{m}=*^{(0)}\left(J^{(0)} \wedge c_{3}^{(0)}\right), \quad *^{(0)}\left(c_{3}^{(0)} \wedge \omega_{i}^{(0)}\right)=-2 Z_{m \bar{n}} \omega_{i}^{(0)} \bar{n} m,
\end{aligned}
$$

and yields the fourth Chern-form $c_{4}^{(0)}$ by contraction with the Riemann tensor as

$$
Z_{m \bar{m} n \bar{n}} R^{(0) \bar{m} m \bar{n} n}=*^{(0)} c_{4}^{(0)} .
$$

We note that $Y_{i j m \bar{n}}$ is also related to $Z_{m \bar{m} n \bar{n}}$ upon integration as

$$
\int_{Y_{4}} Y_{i j m}{ }^{m} *^{(0)} 1=-\frac{1}{6} \int_{Y_{4}}\left(i Z_{m \bar{n}} \omega_{i}^{(0) \bar{r} m} \omega_{j}^{(0) \bar{n}} \bar{r}+2 Z_{m \bar{n} r \bar{s}} \omega_{i}^{(0) \bar{n} m} \omega_{j}^{(0) \bar{s} r}\right) *^{(0)} 1
$$

where the right hand side represents the same linear combination that will be relevant in (B.6). We will see in the next section that the three-dimensional effective action contains the various contractions of $Z_{m \bar{m} n \bar{n}}$. Interestingly, the analog quantity on Calabi-Yau threefolds has played a key role in the analysis of [21]. 


\section{The three-dimensional effective action}

In this section we derive the three-dimensional effective action for the scalar and vector fields introduced in section 3. The kinetic terms for the Kähler structure deformations and vector fields will be discussed. In a flux background also Chern-Simons terms are induced and will be included in our analysis. ${ }^{2}$ We also study a non-trivial field-dependent scaling symmetry of the kinetic terms, which involves a rescaling of the warp-factor. Some of the technical details of the performed reduction are supplemented in appendix B.

Having identified the background of eleven-dimensional action in section 2 and a set of perturbations in section 3 we are now in a position to derive the three-dimensional effective action using a dimensional reduction. To systematically approach this task we will consider an expansion up to second order in the scalar fluctuations $\delta v^{i}$ and vectors $A^{i}$. Furthermore, we will restrict our analysis to terms with only two external space derivatives and only retain terms up to order $\alpha^{2}$.

For the convenience of the reader we begin by summarising the full ansatz that we will use in the reduction. The perturbed eleven-dimensional metric takes the form

$$
\begin{aligned}
d \hat{s}^{2}= & e^{-\frac{512}{3} \alpha^{2}\left(Z\left|+\partial_{i} Z\right| \delta v^{i}+\frac{1}{2} \partial_{i} \partial_{j} Z \mid \delta v^{i} \delta v^{j}\right)}\left[e^{-2 \alpha^{2}\left(W^{(2)}\left|+\partial_{i} W^{(2)}\right| \delta v^{i}+\frac{1}{2} \partial_{i} \partial_{j} W^{(2)} \mid \delta v^{i} \delta v^{j}\right)} g_{\mu \nu} d x^{\mu} d x^{\nu}\right. \\
& +2 e^{\alpha^{2}\left(W^{(2)}\left|+\partial_{i} W^{(2)}\right| \delta v^{i}+\frac{1}{2} \partial_{i} \partial_{j} W^{(2)} \mid \delta v^{i} \delta v^{j}\right)}\left(g_{m \bar{n}}^{(0)}+\omega_{i}^{(0)}{ }_{m \bar{n}} d v^{i}\right. \\
& \left.\left.+\alpha^{2} \partial_{m} \partial_{\bar{n}}\left(\tilde{F}\left|+\rho_{i}^{(\mathrm{s})} \delta v^{i}+\partial_{i} \tilde{F}\right| \delta v^{i}+\frac{1}{2} \partial_{i} \partial_{j} \tilde{F} \mid \delta v^{i} \delta v^{j}\right)\right) d y^{m} d y^{\bar{n}}\right]+\mathcal{O}\left(\alpha^{3}\right)+\mathcal{O}\left(\delta v^{i 3}\right),
\end{aligned}
$$

while the perturbed M-theory four-form field strength is given by

$$
\begin{aligned}
\hat{G}= & \alpha G^{(1)}+F^{i} \wedge \omega_{i}^{(0)}+\alpha^{2} F^{i} \wedge \partial \bar{\partial} \rho_{i}^{(\mathrm{v})} \\
& +*_{3} 1 \wedge d e^{-3 \alpha^{2}\left(W^{(2)}\left|+\partial_{i} W^{(2)}\right| \delta v^{i}+\frac{1}{2} \partial_{i} \partial_{j} W^{(2)} \mid \delta v^{i} \delta v^{j}\right)}+\mathcal{O}\left(\alpha^{3}\right)+\mathcal{O}\left(\delta v^{i 3}\right) .
\end{aligned}
$$

The rather involved form of this ansatz reflects the fact that the quantities present are expanded in both $\alpha$ and $\delta v^{i}$. Recall that the symbol $\mid$ means evaluation at $\delta v^{i}=0, \partial_{i}$ are derivatives with respect to $v^{i}$, and $\partial_{m}, \partial_{\bar{n}}$ are space-time derivatives in the lowest-order complex structure of the internal manifold.

The quantities $Z\left|, \partial_{i} Z\right|, \partial_{i} \partial_{j} Z \mid$ are directly evaluated by using the definition of $Z$ given in (3.16). Similarly one proceeds with the derivatives of $\tilde{F}=*(J \wedge J \wedge F)$ given in (2.19). In contrast, since the warp-factor $W^{(2)}$ is only known as a solution to the warpfactor equation (2.20) one would have to apply (3.11) and (3.12) to determine $\partial_{i} W^{(2)} \mid$ and $\partial_{i} \partial_{j} W^{(2)} \mid$. It turns out to be sufficient, however, to keep $\partial_{i} W^{(2)} \mid$ and $\partial_{i} \partial_{j} W^{(2)} \mid$ throughout the analysis. Remarkably, we will find that all contributions involving $\partial_{i} \partial_{j} W^{(2)} \mid$ precisely cancel, while the first derivatives $\partial_{i} W^{(2)} \mid$ appear in the correct way to ensure the presence of a $v^{i}$-dependent scaling symmetry involving the warp-factor. Before turning to the derivation, let us also note that one may include compensators in the effective action along the lines of the discussion presented in $[24,27,40]$. However these do not change the effective action at the studied order.

\footnotetext{
${ }^{2}$ Note that these terms are topological in nature and key in the study of chiral F-theory spectra and anomalies [38, 39].
} 
In this subsection we only discuss the kinetic terms that are present in the reduction. The reduction process is quite lengthy and makes use of the intermediate results listed in appendix B. One inserts the ansatz (4.1), (4.2) into the eleven-dimensional action (2.3). The dimensional reduction requires numerous partial integrations and uses multiple Schouten and Bianchi identities, which was only possible by using a computer algorithm. Our goal was to represent all three-dimensional terms using the higher-curvature tensor $Z_{m \bar{m} n \bar{n}}$ introduced in (3.13). Combining all terms of the computation we find the action

$$
S_{\mathrm{kin}}=S_{\mathrm{kin}}^{(0)}+\alpha S_{\mathrm{CS}}^{(1)}+\alpha^{2} S_{\mathrm{kin}}^{(2)},
$$

where at zeroth order one has

$$
\begin{aligned}
S_{\mathrm{kin}}^{(0)}=\frac{1}{2 \kappa_{11}} \int_{\mathcal{M}_{3}}[ & \Omega^{(0)} R * 1+d \delta v^{i} \wedge * d \delta v^{j} \int_{Y_{4}}\left(\frac{1}{2} \omega_{i m \bar{n}}^{(0)} \omega_{j}^{(0) \bar{n} m}-\omega_{i m}^{(0) m} \omega_{j n}^{(0) n}\right) *^{(0)} 1 \\
& \left.+\frac{1}{2} F^{i} \wedge * F^{j} \int_{Y_{4}} \omega_{i m \bar{n}}^{(0)} \omega_{j}^{(0)} \bar{n} m *^{(0)} 1\right]
\end{aligned}
$$

while at first order one finds the Chern-Simons terms

$$
S_{\mathrm{CS}}^{(1)}=\frac{1}{2 \kappa_{11}} \int_{\mathcal{M}_{3}} \Theta_{i j} A^{j} \wedge F^{i}, \quad \Theta_{i j}=\frac{1}{2} \alpha \int_{Y_{4}} \omega_{i}^{(0)} \wedge \omega_{j}^{(0)} \wedge G^{(1)},
$$

and at second order

$$
\begin{gathered}
S_{\text {kin }}^{(2)}=\frac{1}{2 \kappa_{11}} \int_{\mathcal{M}_{3}}\left[\Omega^{(2)} R * 1+d \delta v^{i} \wedge * d \delta v^{j} \int_{Y_{4}}\left(3 i \partial_{i} W^{(2)} \mid \omega_{j m}^{(0) m}+3 W^{(2)}\left(\frac{1}{2} \omega_{i m \bar{n}}^{(0)} \omega_{j}^{(0) \bar{n} m}-\omega_{i m}^{(0) m} \omega_{j n}^{(0) n}\right)\right.\right. \\
\left.-768 Z \omega_{i m}^{(0) m} \omega_{j n}^{(0) n}+3072 i Z_{m \bar{n}} \omega_{i}^{(0) \bar{n} m} \omega_{j s}^{(0) s}+3072 Z_{m \bar{n}} r \bar{s} \omega_{i}^{(0) \bar{n} m} \omega_{j}^{(0) \bar{s} r}\right) *{ }^{(0)} 1 \\
+F^{i} \wedge * F^{j} \int_{Y_{4}}\left(\left(\frac{3}{2} W^{(2)}+256 Z\right) \omega_{i m \bar{n}}^{(0)} \omega_{j}^{(0) \bar{n} m}+192\left(-7+a_{1}\right) i Z_{m \bar{n}} \omega_{i}^{(0) \bar{r} m} \omega_{j}^{(0) \bar{n}} \overline{\bar{r}}\right. \\
\left.\left.+384\left(1+a_{1}\right) Z_{m \bar{n} r \bar{s}} \omega_{i}^{(0) \bar{n} m} \omega_{j}^{(0) \bar{s} r}\right) *^{(0)} 1\right] .
\end{gathered}
$$

Here we have abbreviated

$$
\begin{aligned}
& \Omega^{(0)}= \int_{Y_{4}}\left[1+i \delta v^{i} \omega_{i m}^{(0) m}+\frac{1}{2} \delta v^{i} \delta v^{j}\left(\omega_{i m \bar{n}}^{(0)} \omega_{j}^{(0)} \bar{n} m-\omega_{i m}^{(0)} m \omega_{j n}^{(0)} n\right)\right] *^{(0)} 1 \\
& \Omega^{(2)}=\int_{Y_{4}}\left[3 W^{(2)}+3 \delta v^{i}\left(\partial_{i} W^{(2)} \mid+i \omega_{i m}^{(0)} m W^{(2)}\right)+\delta v^{j} \delta v^{i}\left(\frac{3}{2} \partial_{i} \partial_{j} W^{(2)} \mid\right.\right. \\
&\left.\left.+3 i \omega_{i m}^{(0) m} \partial_{j} W^{(2)} \mid+\frac{3}{2} W^{(2)}\left(\omega_{i m \bar{n}}^{(0)} \omega_{j}^{(0)} \bar{n} m-\omega_{i m}^{(0)} m \omega_{j n}^{(0)} n\right)\right)\right] *^{(0)} 1 .
\end{aligned}
$$

A few comments are in order. Firstly, we show in appendix B that among all the terms in (2.10) only $\mathcal{A}, \mathcal{Z}_{1}$ an $\mathcal{Z}_{2}$ contribute, while $\mathcal{Z}_{3}$ to $\mathcal{Z}_{6}$ vanish identically. This implies that the result should depend on two unknown parameters $a_{1}, a_{2}$ that appear in (2.10). It turns out that for the choice $a_{1}=a_{2}$ the result simplifies significantly and only depends on $Z_{m \bar{m} n \bar{n}}$ as is equally true for the reduction of all other term in the eleven-dimensional action (2.3). We therefore have chosen $a_{1}=a_{2}$ in (4.6). Secondly, we note that, as already mentioned before, the scalar functions $\tilde{F}, \rho^{(\mathrm{s})}{ }_{i}$ and $\rho^{(\mathrm{v})}{ }_{i}$ have totally dropped out of this expression. This justifies the use of $\operatorname{dim}\left(H^{1,1}\left(Y_{4}\right)\right)$ deformations $\delta v^{i}$ and vectors $A^{i}$. 
The action (4.6) still depends on $\partial_{i} \partial_{j} W^{(2)}$, however, only through the coefficient of the three-dimensional Einstein-Hilbert term. We now wish to Weyl rescale this action to bring it to the Einstein frame and show that this dependence actually drops. From (A.5) one finds that one needs to redefine the external metric by $g_{\mu \nu} \rightarrow g_{\mu \nu}^{\prime}=\Omega^{-2} g_{\mu \nu}$ for

$$
\Omega=\Omega^{(0)}+\alpha^{2} \Omega^{(2)} .
$$

Performing the Weyl rescaling we find that the kinetic terms displayed in (4.4) and (4.6) become

$$
\begin{gathered}
S_{\text {kin }}^{(0)}=\frac{1}{2 \kappa_{11}} \int_{\mathcal{M}_{3}}\left[R * 1+d \delta v^{i} \wedge * d \delta v^{j} \frac{1}{\mathcal{V}_{0}} \int_{Y_{4}}\left(\frac{1}{2} \omega_{i m \bar{n}}^{(0)} \omega_{j}^{(0) \bar{n} m}+\omega_{i m}^{(0)} \omega_{j n}^{(0) n}\right) *^{(0)} 1\right. \\
\left.+F^{i} \wedge * F^{j} \frac{\mathcal{V}_{0}}{2} \int_{Y_{4}} \omega_{i m \bar{n}}^{(0)} \omega_{j}^{(0)} \bar{n} m *^{(0)} 1\right]
\end{gathered}
$$

and

$$
\begin{aligned}
S_{\mathrm{kin}}^{(2)} & =\frac{1}{2 \kappa_{11}} \int_{\mathcal{M}_{3}}\left[d \delta v ^ { i } \wedge * d \delta v ^ { j } \left(\frac { 1 } { \mathcal { V } _ { 0 } } \int _ { Y _ { 4 } } \left(-9 i \partial_{i} W^{(2)}\left|\omega_{j m}^{(0)} m+\frac{3}{2} W^{(2)}\right| \omega_{i m \bar{n}}^{(0)} \omega_{j}^{(0) \bar{n} m}\right.\right.\right. \\
& \left.-768 Z \omega_{i m}^{(0) m} \omega_{j n}^{(0) n}+3072 i Z_{m \bar{n}} \omega_{i}^{(0) \bar{n} m} \omega_{i s}^{(0) s}+3072 Z_{m \bar{n} r \bar{s}} \omega_{i}^{(0) \bar{n} m} \omega_{j}^{(0) \bar{s} r}\right) *^{(0)} 1 \\
& \left.-\frac{1}{\mathcal{V}_{0}^{2}} \int_{Y_{4}} \frac{3}{2} W^{(2)} \mid *^{(0)} 1 \int_{Y_{4}} \omega_{i m \bar{n}}^{(0)} \omega_{j}^{(0) \bar{n} m} *^{(0)} 1\right) \\
& +F^{i} \wedge * F^{j}\left(\mathcal { V } _ { 0 } \int _ { Y _ { 4 } } \left(\left(\frac{3}{2} W^{(2)} \mid+256 Z\right) \omega_{i m \bar{n}}^{(0)} \omega_{j}^{(0) \bar{n} m}+192\left(-7+a_{1}\right) i Z_{m \bar{n}} \omega_{i}^{(0) \bar{r} m} \omega_{j}^{(0) \bar{n}} \overline{\bar{r}}\right.\right. \\
& \left.\left.\left.+384\left(1+a_{1}\right) Z_{m \bar{n} r \bar{s}} \omega_{i}^{(0) \bar{n} m} \omega_{j}^{(0) \bar{r} r}\right) *^{(0)} 1+\int_{Y_{4}} \frac{3}{2} W^{(2)} \mid *^{(0)} 1 \int_{Y_{4}} \omega_{i m \bar{n}}^{(0)} \omega_{j}^{(0) \bar{n} m} *^{(0)} 1\right)\right],
\end{aligned}
$$

where here we have introduced the zeroth-order volume

$$
\mathcal{V}_{0}=\int_{Y_{4}} *^{(0)} 1
$$

The warp-factor dependence can be nicely captured by introducing the warped volume and warped metric

$$
\mathcal{V}_{W}=\int_{Y_{4}} e^{3 \alpha^{2} W^{(2)}} *^{(0)} 1, \quad G_{i j}^{W}=\frac{1}{2 \mathcal{V}_{W}} \int_{Y_{4}} e^{3 \alpha^{2} W^{(2)}} \omega_{i}^{(0)} \wedge *^{(0)} \omega_{j}^{(0)},
$$

which at zeroth order in $\alpha$ reduce to $\mathcal{V}_{0}$ and $G_{i j}=\frac{1}{2 \mathcal{V}_{0}} \int_{Y_{4}} \omega_{i}^{(0)} \wedge *^{(0)} \omega_{j}^{(0)}$. We also introduce

$$
K_{i}^{W}=i \mathcal{V}_{W} \omega_{i m}^{(0) m}+\frac{9}{2} \alpha^{2} \int_{Y_{4}} \partial_{i} W^{(2)} \mid *^{(0)} 1
$$

which at lowest order simply reduces to $K_{i}=i \mathcal{V}_{0} \omega_{i m}^{(0)} m=\frac{1}{3 !} \int_{Y_{4}} \omega_{i}^{(0)} \wedge J^{(0)} \wedge J^{(0)} \wedge J^{(0)}$. With these definitions one rewrites the action (4.3) for all kinetic terms into the form $S_{\text {kin }}=\frac{1}{2 \kappa_{11}} \int_{\mathcal{M}_{3}}\left[R * 1-\left(G_{i j}^{W}+\mathcal{V}_{W}^{-2} K_{i}^{W} K_{j}^{W}\right) d v^{i} \wedge * d v^{j}-\mathcal{V}_{W}^{2} G_{i j}^{W} F^{i} \wedge * F^{j}+\Theta_{i j} A^{i} \wedge F^{i}\right.$ 


$$
\begin{aligned}
& -d v^{i} \wedge * d v^{j} \frac{\alpha^{2}}{\mathcal{V}_{0}} \int_{Y_{4}}\left(768 Z \omega_{i m}^{(0) m} \omega_{j n}^{(0) n}-3072 i Z_{m \bar{n}} \omega_{i}^{(0) \bar{n} m} \omega_{j s}^{(0) s}-3072 Z_{m \bar{n} r \bar{s}} \omega_{i}^{(0) \bar{n} m} \omega_{j}^{(0) \bar{s} r}\right) *^{(0)} 1 \\
& +F^{i} \wedge * F^{j} \alpha^{2} \mathcal{V}_{0} \int_{Y_{4}}\left(256 Z \omega_{i m \bar{n}}^{(0)} \omega_{j}^{(0) \bar{n} m}+192\left(-7+a_{1}\right) i Z_{m \bar{n}} \omega_{i}^{(0) \bar{r} m} \omega_{j}^{(0) \bar{n}} \overline{\bar{r}}\right. \\
& \left.\left.+384\left(1+a_{1}\right) Z_{m \bar{n} r \bar{s}} \omega_{i}^{(0) \bar{n} m} \omega_{j}^{(0) \bar{s} r}\right) *^{(0)} 1\right]
\end{aligned}
$$

where we have replaced $d \delta v^{i}$ directly with $d v^{i}$. Expanding to order $\alpha^{2}$ one indeed recovers the above result.

It is interesting to observe that the three-dimensional effective action permits a scaling symmetry involving the rescaling of the warp-factor. We begin by noting that the elevendimensional background ansatz given in subsection 2.2 has a symmetry under which

$$
W^{(2)} \rightarrow W^{(2)}+\Lambda^{(2)}, \quad g_{m \bar{n}} \rightarrow e^{-\alpha^{2} \Lambda^{(2)}} g_{m \bar{n}}, \quad g_{\mu \nu} \rightarrow e^{2 \alpha^{2} \Lambda^{(2)}} g_{\mu \nu},
$$

for $\Lambda^{(2)}=\Lambda^{(2)}\left(x^{\mu}\right)$. This can be extended to a symmetry of the perturbed background (4.1) and (4.2) by requiring that

$$
v^{i} \rightarrow e^{-\alpha^{2} \Lambda^{(2)}} v^{i}
$$

This then implies that

$$
d v^{i} \rightarrow e^{-\alpha^{2} \Lambda^{(2)}} d v^{i}-\alpha^{2} v^{i} \partial_{j} \Lambda^{(2)} d v^{j},
$$

if we further restrict $\Lambda^{(2)}=\Lambda^{(2)}\left(v^{i}\right)$. When the reduction is performed this the becomes a symmetry of the effective action before the Weyl rescaling to move to the Einstein frame is performed. When the rescaling is performed the value of $\Omega$ in $g_{\mu \nu} \rightarrow g_{\mu \nu}^{\prime}=\Omega^{-2} g_{\mu \nu}$ transforms as $\Omega \rightarrow e^{-\alpha^{2} W^{(2)}} \Omega$ so that the rescaled metric does not transform. The final form of the effective action coming from the dimensional reduction is then invariant under the symmetry

$$
W^{(2)} \rightarrow W^{(2)}+\Lambda^{(2)}, \quad v^{i} \rightarrow e^{-\alpha^{2} \Lambda^{(2)}} v^{i} .
$$

We note that the $\partial_{i} W^{(2)}$ terms in the $\delta v^{i}$ kinetic terms are key to ensuring the symmetry of the action for $\Lambda^{(2)}$ as a function of $v^{i}$, as they covariantize the derivatives which appear in the reduction. Indeed, this symmetry can be made manifest by introducing a covariant derivative for $v^{i}$. Furthermore we note that if we make the choice $a_{1}=7$ then using the definitions,

$$
\begin{aligned}
\mathcal{G}_{i j}^{T}= & G_{i j}^{W}+256 \frac{1}{\mathcal{V}_{0}^{2}} \int_{Y_{4}} Z *^{(0)} 1 \int_{Y_{4}} \omega_{i}^{(0)} m \bar{n} \omega_{j}^{(0) \bar{n} m} *^{(0)} 1 \\
& -256 \frac{1}{\mathcal{V}_{0}} \int_{Y_{4}}\left[Z \omega_{i}^{(0)} m \bar{n} \omega_{j}^{(0) \bar{n} m}+12 Z_{m \bar{n} r \bar{s}} \omega_{j}^{(0)} \bar{n} m \omega_{i}^{(0) \bar{s} r}\right] *^{(0)} 1 \\
\mathcal{K}_{i}^{T}= & K_{i}+\alpha^{2} \int_{Y_{4}}\left[\frac{1}{3 !}\left(3 W^{(2)}-128 Z\right) J^{(0)} \wedge J^{(0)} \wedge J^{(0)} \wedge \omega_{i}^{(0)}-1536 Z_{m \bar{n}} \omega_{i}^{(0) \bar{n} m} *^{(0)} 1\right], \\
\mathcal{V}_{T}= & \mathcal{V}_{W}+\alpha^{2} 256 \int_{Y_{4}} Z *^{(0)} 1, \quad D v^{i}=d v^{i}+\alpha^{2} v^{i} d v^{j} \int_{Y_{4}} \partial_{j} W^{(2)} *^{(0)} 1
\end{aligned}
$$


the action takes the simple form ${ }^{3}$

$$
S_{\text {kin }}=\frac{1}{2 \kappa_{11}} \int_{\mathcal{M}_{3}}\left[R * 1-\left(\mathcal{G}_{i j}^{T}+\mathcal{V}_{T}^{-2} \mathcal{K}_{i}^{T} \mathcal{K}_{j}^{T}\right) D v^{i} \wedge * D v^{j}-\mathcal{V}_{T}^{2} \mathcal{G}_{i j}^{T} F^{i} \wedge * F^{j}+\Theta_{i j} A^{i} \wedge F^{i}\right] .
$$

Where now it is clear that under (4.18)

$$
\mathcal{G}_{i j}^{T} \rightarrow e^{2 \alpha^{2} \Lambda^{(2)}} \mathcal{G}_{i j}^{T}, \quad \mathcal{V}_{T} \rightarrow e^{-\alpha^{2} \Lambda^{(2)}} \mathcal{V}_{T}, \quad \mathcal{K}_{i}^{T} \rightarrow \mathcal{K}_{i}^{T}, \quad D v^{i} \rightarrow e^{-\alpha^{2} \Lambda^{(2)}} D v^{i}
$$

so that the action (4.21) is invariant.

Finally, let us briefly discuss the potential of the three-dimensional effective theory. It is well-known that it contains a flux-dependent part given by [36, 37]

$$
S_{\text {pot }}=\frac{\alpha^{2}}{4 \kappa_{11}} \int_{\mathcal{M}_{3}} * 1 \int_{Y_{4}}\left(G^{(1)} \wedge * G^{(1)}-G^{(1)} \wedge G^{(1)}\right),
$$

in which the internal Hodge star is evaluated in the perturbed zeroth-order metric (3.8) which sees the full $v^{i}$. This term is responsible for imposing self-duality of $G^{(1)}$ in the vacuum. With our restriction to Kähler deformations this implies that $G^{(1)}$ remains primitive with respect to the perturbed metric (3.8) for massless fluctuations, i.e. the ones satisfying (3.9). We can easily see that warping or higher-curvature corrections which multiply this result will yield corrections that are higher than order $\alpha^{2}$ and thus cannot be reliably analyzed using our ansatz. Furthermore, we propose that at order $\alpha^{2}$ there are no terms added to (4.23) that are only dependent on the warping and internal space higher-curvature terms, as the background we analyse is invariant under the perturbations we consider as long as $G^{(1)}$ remains self-dual. This will be demonstrated in [20].

Let us close by noting that in a next step one has to bring the action into standard $\mathcal{N}=2$ form and determine a kinetic potential and the correct $\mathcal{N}=2$ coordinates. This will be done in the second part of this paper [20].

\section{A Conventions, definitions, and identities}

In this work we denote the eleven-dimensional space indices by capital Latin letters $M, N, R=0, \ldots, 10$, the external ones by $\mu, \nu=0,1,2$, and the internal complex ones by $m, n, p=1, \ldots, 4$ and $\bar{m}, \bar{n}, \bar{p}=1, \ldots, 4$. Eleven-dimensional quantities for which the indices are raised and lower with the total space metric carry a hat, for example the M-theory three-form is denoted by $\hat{G}$. Furthermore, the convention for the totally antisymmetric tensor in Lorentzian space in an orthonormal frame is $\epsilon_{012 \ldots 10}=\epsilon_{012}=+1$. The epsilon tensor in $d$ dimensions then satisfies

$$
\left.\epsilon^{R_{1} \cdots R_{p} N_{1} \ldots N_{d-p}} \epsilon_{R_{1} \ldots R_{p} M_{1} \ldots M_{d-p}}=(-1)^{s}(d-p) ! p ! \delta^{N_{1}}{ }_{\left[M_{1}\right.} \ldots \delta^{N_{d-p}} M_{d-p}\right]
$$

\footnotetext{
${ }^{3}$ Note that in making this match we have used that$$
\int_{\mathcal{M}_{3}} d v^{i} \wedge * d v^{j} \frac{1}{\mathcal{V}_{0}} \int_{Y_{4}} Z \omega_{i}^{(0)} \wedge *^{(0)} \omega_{j}^{(0)}=\int_{\mathcal{M}_{3}} d v^{i} \wedge * d v^{j} \frac{1}{\mathcal{V}_{0}^{2}} \int_{Y_{4}} Z^{(0)} * 1 \int_{Y_{4}} \omega_{i}^{(0)} \wedge *^{(0)} \omega_{j}^{(0)}
$$$$
\int_{\mathcal{M}_{3}} d v^{i} \wedge * d v^{j} \frac{1}{\mathcal{V}_{0}} \int_{Y_{4}} W \omega_{i}^{(0)} \wedge *^{(0)} \omega_{j}^{(0)}=\int_{\mathcal{M}_{3}} d v^{i} \wedge * d v^{j} \frac{1}{\mathcal{V}_{0}^{2}} \int_{Y_{4}} W *^{(0)} 1 \int_{Y_{4}} \omega_{i}^{(0)} \wedge *^{(0)} \omega_{j}^{(0)}
$$

which can be demonstrated by taking using integration by parts in the external space. 
where $s=0$ if the metric has Riemannian signature and $s=1$ for a Lorentzian metric.

We adopt the following conventions for the Christoffel symbols and Riemann tensor

$$
\begin{aligned}
\Gamma^{R}{ }_{M N} & =\frac{1}{2} g^{R S}\left(\partial_{M} g_{N S}+\partial_{N} g_{M S}-\partial_{S} g_{M N}\right), & R_{M N} & =R^{R}{ }_{M R N}, \\
R^{M}{ }_{N R S} & =\partial_{R} \Gamma^{M}{ }_{S N}-\partial_{S} \Gamma^{M}{ }_{R N}+\Gamma^{M}{ }_{R T} \Gamma^{T}{ }_{S N}-\Gamma^{M}{ }_{S T} \Gamma^{T}{ }_{R N}, & R & =R_{M N} g^{M N},
\end{aligned}
$$

with equivalent definitions on the internal and external spaces.

The terms $\hat{t}_{8} \hat{t}_{8} \hat{R}^{4}$ and $\hat{t}_{8} \hat{t}_{8} \hat{G}^{2} \hat{R}^{3}$ in (2.4) and (2.5) require the definition

$$
\begin{aligned}
\hat{t}_{8}^{N_{1} \ldots N_{8}}= & \frac{1}{16}\left(-2\left(\hat{g}^{N_{1} N_{3}} \hat{g}^{N_{2} N_{4}} \hat{g}^{N_{5} N_{7}} \hat{g}^{N_{6} N_{8}}+\hat{g}^{N_{1} N_{5}} \hat{g}^{N_{2} N_{6}} \hat{g}^{N_{3} N_{7}} \hat{g}^{N_{4} N_{8}}+\hat{g}^{N_{1} N_{7}} \hat{g}^{N_{2} N_{8}} \hat{g}^{N_{3} N_{5}} \hat{g}^{N_{4} N_{6}}\right)\right. \\
& +8\left(\hat{g}^{N_{2} N_{3}} \hat{g}^{N_{4} N_{5}} \hat{g}^{N_{6} N_{7}} \hat{g}^{N_{8} N_{1}}+\hat{g}^{N_{2} N_{5}} \hat{g}^{N_{6} N_{3}} \hat{g}^{N_{4} N_{7}} \hat{g}^{N_{8} N_{1}}+\hat{g}^{N_{2} N_{5}} \hat{g}^{N_{6} N_{7}} \hat{g}^{N_{8} N_{3}} \hat{g}^{N_{4} N_{1}}\right) \\
& \left.-\left(N_{1} \leftrightarrow N_{2}\right)-\left(N_{3} \leftrightarrow N_{4}\right)-\left(N_{5} \leftrightarrow N_{6}\right)-\left(N_{7} \leftrightarrow N_{8}\right)\right) .
\end{aligned}
$$

In order to discuss the term $\hat{s}_{18}$ appearing in (2.6) and (2.10) we introduce the basis

$$
\begin{aligned}
& B_{1}=\hat{R}_{N_{1} N_{2} N_{3} N_{4}} \hat{R}_{N_{5} N_{6} N_{7} N_{8}} \hat{\nabla}^{N_{5}} \hat{G}^{N_{1} N_{7} N_{8}}{ }_{N_{9}} \hat{\nabla}^{N_{3}} \hat{G}^{N_{2} N_{4} N_{6} N_{9}}, \\
& B_{13}=\hat{R}_{N_{1} N_{2} N_{3} N_{4}} \hat{R}_{N_{5}}{ }^{N_{1}}{ }_{N_{6}}{ }^{N_{3}} \hat{\nabla}_{N_{9}} \hat{G}^{N_{2} N_{6}}{ }_{N_{7} N_{8}} \hat{\nabla}^{N_{9}} \hat{G}^{N_{4} N_{5} N_{7} N_{8}} \text {, } \\
& B_{2}=\hat{R}_{N_{1} N_{2} N_{3} N_{4}} \hat{R}_{N_{5} N_{6} N_{7} N_{8}} \hat{\nabla}^{N_{5}} \hat{G}^{N_{1} N_{3} N_{7}}{ }_{N_{9}} \hat{\nabla}^{N_{8}} \hat{G}^{N_{2} N_{4} N_{6} N_{9}}, \\
& B_{14}=\hat{R}_{N_{1} N_{2} N_{3} N_{4}} \hat{R}_{N_{5}}{ }^{N_{1}}{ }_{N_{6}}{ }^{N_{3}} \hat{\nabla}_{N_{9}} \hat{G}^{N_{2} N_{4}}{ }_{N_{7} N_{8}} \hat{\nabla}^{N_{9}} \hat{G}^{N_{5} N_{6} N_{7} N_{8}} \text {, } \\
& B_{3}=\hat{R}_{N_{1} N_{2} N_{3} N_{4}} \hat{R}_{N_{5} N_{6} N_{7} N_{8}} \hat{\nabla}^{N_{5}} \hat{G}^{N_{1} N_{3} N_{7}}{ }_{N_{9}} \hat{\nabla}^{N_{6}} \hat{G}^{N_{2} N_{4} N_{8} N_{9}}, \\
& B_{15}=\hat{R}_{N_{1} N_{2} N_{3} N_{4}} \hat{R}_{N_{5}}{ }^{N_{1}}{ }_{N_{6}}{ }^{N_{3}} \hat{\nabla}^{N_{2}} \hat{G}^{N_{6}}{ }_{N_{7} N_{8} N_{9}} \hat{\nabla}^{N_{5}} \hat{G}^{N_{4} N_{7} N_{8} N_{9}} \text {, } \\
& B_{4}=\hat{R}_{N_{1} N_{2} N_{3} N_{4}} \hat{R}_{N_{5} N_{6} N_{7} N_{8}} \hat{\nabla}_{N_{9}} \hat{G}^{N_{3} N_{4} N_{7} N_{8}} \hat{\nabla}^{N_{6}} \hat{G}^{N_{9} N_{1} N_{2} N_{5}}, \\
& B_{16}=\hat{R}_{N_{1} N_{2} N_{3} N_{4}} \hat{R}_{N_{5}}{ }^{N_{1}}{ }_{N_{6}}{ }^{N_{3}} \hat{\nabla}^{N_{2}} \hat{G}^{N_{4}}{ }_{N_{7} N_{8} N_{9}} \hat{\nabla}^{N_{5}} \hat{G}^{N_{6} N_{7} N_{8} N_{9}} \text {, }
\end{aligned}
$$

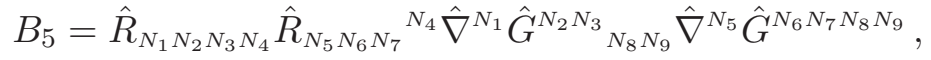

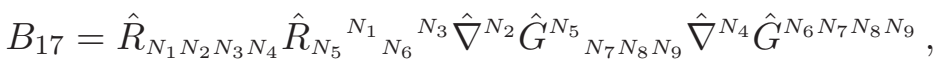

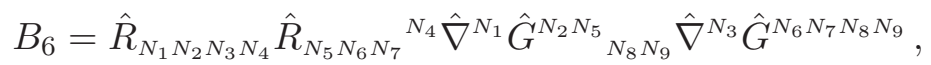

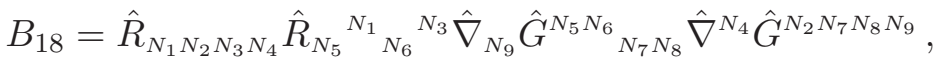

$$
\begin{aligned}
& B_{7}=\hat{R}_{N_{1} N_{2} N_{3} N_{4}} \hat{R}_{N_{5} N_{6} N_{7}}{ }^{N_{4}} \hat{\nabla}^{N_{1}} \hat{G}^{N_{2} N_{5}{ }_{N_{8} N_{9}}} \hat{\nabla}^{N_{7}} \hat{G}^{N_{3} N_{6} N_{8} N_{9}}, \\
& B_{19}=\hat{R}_{N_{1} N_{2} N_{3} N_{4}} \hat{R}_{N_{5} N_{6}}{ }^{N_{3} N_{4}} \hat{\nabla}_{N_{9}} \hat{G}^{N_{1} N_{5}}{ }_{N_{7} N_{8}} \hat{\nabla}^{N_{9}} \hat{G}^{N_{2} N_{6} N_{7} N_{8}}, \\
& B_{8}=\hat{R}_{N_{1} N_{2} N_{3} N_{4}} \hat{R}_{N_{5} N_{6} N_{7}}{ }^{N_{4}} \hat{\nabla}^{N_{1}} \hat{G}^{N_{3} N_{5}}{ }_{N_{8} N_{9}} \hat{\nabla}^{N_{2}} \hat{G}^{N_{6} N_{7} N_{8} N_{9}} \text {, } \\
& B_{20}=\hat{R}_{N_{1} N_{2} N_{3} N_{4}} \hat{R}_{N_{5} N_{6}}{ }^{N_{3} N_{4}} \hat{\nabla}^{N_{1}} \hat{G}^{N_{5}}{ }_{N_{7} N_{8} N_{9}} \hat{\nabla}^{N_{2}} \hat{G}^{N_{6} N_{7} N_{8} N_{9}} \text {, } \\
& B_{9}=\hat{R}_{N_{1} N_{2} N_{3} N_{4}} \hat{R}_{N_{5} N_{6} N_{7}}{ }^{N_{4}} \hat{\nabla}^{N_{1}} \hat{G}^{N_{3} N_{5}}{ }_{N_{8} N_{9}} \hat{\nabla}^{N_{6}} \hat{G}^{N_{2} N_{7} N_{8} N_{9}} \text {, } \\
& B_{21}=\hat{R}_{N_{1} N_{2} N_{3} N_{4}} \hat{R}_{N_{5} N_{6}}{ }^{N_{3} N_{4}} \hat{\nabla}^{N_{1}} \hat{G}^{N_{5}}{ }_{N_{7} N_{8} N_{9}} \hat{\nabla}^{N_{6}} \hat{G}^{N_{2} N_{7} N_{8} N_{9}} \text {, } \\
& B_{10}=\hat{R}_{N_{1} N_{2} N_{3} N_{4}} \hat{R}_{N_{5} N_{6} N_{7}}^{N_{4}} \hat{\nabla}_{N_{9}} \hat{G}^{N_{3} N_{5} N_{7} N_{8}} \hat{\nabla}^{N_{9}} \hat{G}^{N_{1} N_{2} N_{6} N_{8}} \text {, }
\end{aligned}
$$

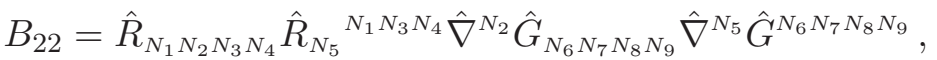

$$
\begin{aligned}
& B_{11}=\hat{R}_{N_{1} N_{2} N_{3} N_{4}} \hat{R}_{N_{5} N_{6} N_{7}}{ }^{N_{4}} \hat{\nabla}_{N_{8}} \hat{G}^{N_{1} N_{2} N_{6}}{ }_{N_{9}} \hat{\nabla}^{N_{9}} \hat{G}^{N_{3} N_{5} N_{7} N_{8}} \text {, } \\
& B_{23}=\hat{R}_{N_{1} N_{2} N_{3} N_{4}} \hat{R}_{N_{5}}{ }^{N_{1} N_{3} N_{4}} \hat{\nabla}_{N_{9}} \hat{G}^{N_{2}}{ }_{N_{6} N_{7} N_{8}} \hat{\nabla}^{N_{9}} \hat{G}^{N_{5} N_{6} N_{7} N_{8}} \text {, } \\
& B_{12}=\hat{R}_{N_{1} N_{2} N_{3} N_{4}} \hat{R}_{N_{5} N_{6} N_{7}}{ }^{N_{4}} \hat{\nabla}^{N_{3}} \hat{G}^{N_{5} N_{6}}{ }_{N_{8} N_{9}} \hat{\nabla}^{N_{7}} \hat{G}^{N_{2} N_{1} N_{8} N_{9}} \text {, } \\
& B_{24}=\hat{R}_{N_{1} N_{2} N_{3} N_{4}} \hat{R}^{N_{1} N_{2} N_{3} N_{4}} \hat{\nabla}_{N_{5}} \hat{G}_{N_{6} N_{7} N_{8} N_{9}} \hat{\nabla}^{N_{6}} \hat{G}^{N_{5} N_{7} N_{8} N_{9}} \text {. }
\end{aligned}
$$

The contributions to $\hat{s}_{18}(\hat{\nabla} \hat{G})^{2} \hat{R}^{2}$ are then formed from the linear combinations described in $(2.10)$. 
We note that performing a Weyl rescaling of the three dimensional external metric with $g_{\mu \nu}^{\prime}=\Omega^{-2} g_{\mu \nu}$ one finds that

$$
\int_{\mathcal{M}_{3}} \Omega R^{\prime} *_{3}^{\prime} 1=\int_{\mathcal{M}_{3}}\left(R *_{3} 1-\frac{2}{\Omega^{2}} \nabla_{\mu} \Omega \nabla^{\mu} \Omega *_{3} 1\right) .
$$

Finally we demonstrate that the 3 d effective action may be simplified by using the intersection structures

$$
\begin{aligned}
& K_{i j k l}=\int_{Y_{4}} \omega_{i}^{(0)} \wedge \omega_{j}^{(0)} \wedge \omega_{k}^{(0)} \wedge \omega_{l}^{(0)}, \quad K_{i j k}=K_{i j k l} v^{l}, \quad K_{i j}=\frac{1}{2} K_{i j k l} v^{k} v^{l}, \\
& K_{i}=\frac{1}{3 !} K_{i j k l} v^{j} v^{k} v^{l}, \quad \quad \mathcal{V}_{0}=\frac{1}{4 !} K_{i j k l} v^{i} v^{j} v^{k} v^{l},
\end{aligned}
$$

\section{B Results of the dimensional reduction}

\section{B.1 Two derivative terms}

The reduction of the lowest order part of the action (2.3) gives the following contribution to the Kinetic terms of the $3 \mathrm{~d}$ theory

$$
\begin{aligned}
& \left.S^{(0)}\right|_{\text {kin }}=\frac{1}{2 \kappa_{11}} \int_{\mathcal{M}_{3}} R * 1 \int_{Y_{4}}\left[e^{\alpha^{2}\left(3 W^{(2)}-768 Z\right)}\left(1+i \delta v^{i} \omega_{i m}^{(0) m}+\frac{1}{2} \delta v^{i} \delta v^{j}\left(\omega_{i m \bar{n}}^{(0)} \omega_{j}^{(0)} \bar{n} m-\omega_{i m}^{(0) m} \omega_{j n}{ }^{n}\right)\right)\right. \\
& +3 \alpha^{2} \delta v^{i} \partial_{i} W^{(2)}\left|+3 i \alpha^{2} \delta v^{i} \delta v^{j} \partial_{(i} W^{(2)}\right| \omega_{j) m}{ }^{m}+\frac{3}{2} \alpha^{2} \delta v^{i} \delta v^{j} \partial_{i} \partial_{j} W^{(2)} \mid \\
& +1536 \alpha^{2} \delta v^{i} Z_{m \bar{n}} \omega_{i}^{(0) \bar{n} m}+i 768 \alpha^{2} Z \delta v^{i} \omega_{i m}^{(0) m} \\
& \left.+384 \alpha^{2} Z \delta v^{i} \delta v^{j} \omega_{i m \bar{n}}^{(0)} \omega_{j}^{(0) \bar{n} m}-384 \alpha^{2} \delta v^{i} \delta v^{j} Z \omega_{i m}^{(0) m} \omega_{j n}^{(0) n}\right] *^{(0)} 1 \\
& +\frac{1}{2 \kappa_{11}} \int_{\mathcal{M}_{3}} d \delta v^{i} \wedge * d \delta v^{j} \int_{Y_{4}}\left[e^{\alpha^{2}\left(3 W^{(2)}-768 Z\right)}\left(\frac{1}{2} \omega_{i m \bar{n}}^{(0)} \omega_{j}^{(0) \bar{n} m}-\omega_{i m}^{(0) m} \omega_{j n}{ }^{n}\right)\right. \\
& \left.+3 i \alpha^{2} \partial_{(i} W^{(2)} \mid \omega_{j) m}^{(0)} m+3072 \alpha^{2} i Z_{m \bar{n}} \omega_{i}^{(0) \bar{n} m} \omega_{j s}^{(0) s}-1536 \alpha^{2} Z \omega_{i m}^{(0) m} \omega_{j n}^{(0) n}\right] *^{(0)} 1 \\
& +\frac{1}{2 \kappa_{11}} \frac{1}{2} \int_{\mathcal{M}_{3}} F^{i} \wedge * F^{j} \int_{Y_{4}} e^{\alpha^{2}\left(3 W^{(2)}-256 Z\right)} \omega_{i m \bar{n}}^{(0)} \omega_{j}^{(0)} \bar{n} m *^{(0)} 1 \\
& +\alpha \frac{1}{2 \kappa_{11}} \int_{\mathcal{M}_{3}} F^{i} \wedge A^{j} \int_{Y_{4}} \frac{1}{2} G^{(1)} \wedge \omega_{i}^{(0)} \wedge \omega_{j}^{(0)} .
\end{aligned}
$$

It is interesting to note that in these terms the value of $\tilde{F}, \rho_{i}^{(\mathrm{s})}$ and $\rho_{i}^{(\mathrm{v})}$ drop out of these expressions as they contribute only internal space total derivatives to the $3 \mathrm{~d}$ effective theory.

\section{B.2 Eight derivative terms}

Let us record the reduction of certain higher derivative terms which are used as intermediate results in deriving the effective action (4.3). These results were computed using the mathematica package xAct and required the use of several internal space total derivative and schouten identities.

$$
\left.\int \hat{t}_{8} \hat{t}_{8} \hat{R}^{4} \hat{*} 1\right|_{\text {kin }}=\frac{1}{2 \kappa_{11}} \int_{\mathcal{M}_{3}} d \delta v^{i} \wedge * d \delta v^{j} \int_{Y_{4}} 384\left(Z \omega_{i m \bar{n}}^{(0)} \omega_{j}^{(0)} \bar{n} m+4 Z_{m \bar{n} r \bar{s}} \omega_{i}^{(0)} \bar{n} m \omega_{j}^{(0) \bar{s} r}\right) *^{(0)} 1,
$$




$$
\begin{aligned}
-\left.\frac{1}{24} \int \hat{\epsilon}_{11} \hat{\epsilon}_{11} \hat{R}^{4} \hat{*} 1\right|_{\mathrm{kin}}= & \frac{1}{2 \kappa_{11}} \int_{\mathcal{M}_{3}} R * 1 \int_{Y_{4}}\left(768 Z-1536 \delta v^{i} Z_{m \bar{n}} \omega_{i}^{(0) \bar{n} m}\right) *^{(0)} 1 \\
& +\frac{1}{2 \kappa_{11}} \int_{\mathcal{M}_{3}} d \delta v^{i} \wedge * d \delta v^{j} \int_{Y_{4}} 1536 Z_{m \bar{n} r \bar{s}} \omega_{i}^{(0) \bar{n} m} \omega_{j}^{(0) \bar{s} r} *^{(0)} 1 \\
\left.\int_{Y_{4}} 3^{2} 2^{13} \hat{C} \wedge \hat{X}_{8}\right|_{\text {kin }}= & 0 .
\end{aligned}
$$

Similarly we note that the reduction of the $\hat{G}^{2} \hat{R}^{3}$ terms uses the identities

$$
\begin{aligned}
-\left.\int \hat{t}_{8} \hat{t}_{8} \hat{G}^{2} \hat{R}^{3} \hat{*} 1\right|_{\mathrm{kin}}= & \frac{1}{2 \kappa_{11}^{2}} 384 \int_{\mathcal{M}_{3}} F^{i} \wedge * F^{j} \int_{Y_{4}}\left[Z \omega_{i m \bar{n}}^{(0)} \omega_{j}^{(0) \bar{n} m}\right. \\
& \left.-4 i Z_{m \bar{n}} \omega_{i}^{(0) \bar{r} m} \omega_{j}^{(0) \bar{n}} \overline{\bar{r}}-4 Z_{m \bar{n} r \bar{s}} \omega^{(0) \bar{n} m}{ }_{i}^{(0) \bar{s} r} \underset{j}{ }\right] *^{(0)} 1, \\
-\left.\frac{1}{96} \int \hat{\epsilon}_{11} \hat{\epsilon}_{11} \hat{G}^{2} \hat{R}^{3} \hat{*} 1\right|_{\mathrm{kin}}= & \frac{1}{2 \kappa_{11}^{2}} 1536 \int_{\mathcal{M}_{3}} F^{i} \wedge * F^{j} \int_{Y_{4}} Z_{m \bar{n} r \bar{s}} \omega_{i}^{(0) \bar{n} m} \omega_{j}^{(0) \bar{s} r} *^{(0)} 1 .
\end{aligned}
$$

Finally reducing the $(\hat{\nabla} \hat{G})^{2} \hat{R}^{2}$ terms in $(2.3)$ gives

$$
\begin{aligned}
& \left.\int \hat{s}_{18}(\hat{\nabla} \hat{G})^{2} \hat{R}^{2} \hat{*} 1\right|_{\text {kin }}=\frac{1}{2 \kappa_{11}^{2}} \int_{\mathcal{M}_{3}} \frac{1}{2} F^{i} \wedge * F^{j} \int_{Y_{4}}\left[-96\left(1+a_{2}\right) \omega_{i}^{(0) \bar{m} n} \omega_{j}^{(0) \bar{r} r} R^{(0) \bar{s}} \overline{\bar{r} n}{ }^{s} R^{(0) \bar{t}}{ }_{\bar{s} r}^{t} R_{s \bar{m} t \bar{t}}^{(0)}\right. \\
& -48\left(2+a_{1}+a_{2}\right) \omega_{i}^{(0) \bar{m} n} \omega_{j}^{(0) \bar{r} r} R^{(0) \bar{s} s}{ }_{t}^{u} R_{n}^{(0)}{ }_{\bar{m} r}{ }^{t} R_{s \bar{r} u \bar{s}}^{(0)}+48\left(1+a_{1}\right) \omega_{i}^{(0) \bar{m} n} \omega_{j}^{(0) \bar{r} r} R^{(0) \bar{s}}{ }_{\bar{m} r}{ }^{s} R^{(0) \bar{t}}{ }_{\bar{r} n}{ }^{t} R_{s \bar{s} t \bar{t}}^{(0)} \\
& +48\left(1+a_{2}\right) \omega_{i}^{(0) \bar{m} n} \omega_{j}^{(0) \bar{r} r} R^{(0) \bar{s}}{ }_{\bar{m} n}{ }^{s} R^{(0) \bar{t}}{ }_{\bar{r} r}{ }^{t} R_{s \bar{s} \bar{t}}^{(0)}-48\left(2+a_{1}+a_{2}\right) \omega_{i}^{(0) \bar{m} n} \omega_{j}^{(0) \bar{r} r} R^{(0) \bar{s}}{ }_{\bar{m} n \bar{r}} R^{(0) \bar{t} s}{ }_{r}^{t} R_{s \bar{s} t \bar{t}}^{(0)} \\
& +24\left(1+a_{1}\right) \omega_{i}^{(0) \bar{m} n} \omega_{j}^{(0) \bar{r} r} R^{(0) \bar{s} s \bar{t} t} R^{(0)}{ }_{n \bar{m} r \bar{r}} R_{s \bar{s} t \bar{t}}^{(0)}+48\left(1+a_{2}\right) \omega_{i}^{(0) \bar{m} n} \omega_{j}^{(0) \bar{r} r} R^{(0) \bar{s} s}{ }_{n}{ }^{t} R^{(0)}{ }_{r \bar{s} s}{ }^{u} R_{t \bar{m} u \bar{r}}^{(0)}
\end{aligned}
$$

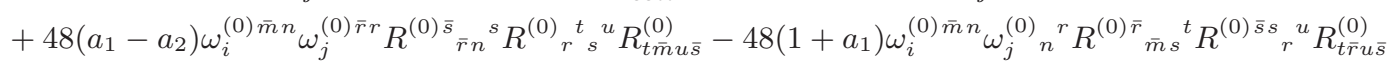

$$
\begin{aligned}
& +48\left(1+a_{1}\right) \omega_{i}^{(0) \bar{m} n} \omega_{j}^{(0)}{ }_{n}^{r} R^{(0) \bar{r}}{ }_{\bar{m} r}{ }^{s} R^{(0) \bar{s} t}{ }_{s}{ }^{u} R_{t \bar{r} u \bar{s}}^{(0)}+48\left(1+a_{2}\right) \omega_{i}^{(0) \bar{m} n} \omega_{j}^{(0) \bar{r} r} R^{(0) \bar{s}}{ }_{\bar{m} s}{ }^{t} R^{(0)}{ }_{n}{ }^{s}{ }_{r}{ }^{u} R_{t \bar{r} u \bar{s}}^{(0)} \\
& +96\left(1+a_{2}\right) \omega_{i}^{(0) \bar{m} n} \omega_{j}^{(0) \bar{r} r} R^{(0) \bar{s}} \bar{m} n{ }^{s} R^{(0)}{ }_{r}{ }^{t}{ }_{s}^{u}{ }^{u} R_{t \bar{r} u \bar{s}}^{(0)}-48\left(1+a_{1}\right) \omega_{i}^{(0) \bar{m} n} \omega_{j}^{(0)}{ }_{n}^{r} R^{(0) \bar{r} s}{ }_{r}^{t} R^{(0)}{ }_{s}{ }^{u}{ }_{t}^{v} R_{u \bar{m} v \bar{r}}^{(0)} \\
& \left.+48\left(1+a_{2}\right) \omega_{i}^{(0) \bar{m} n} \omega_{j}^{(0) \bar{r} r} R_{n}^{(0)}{ }_{n}^{s}{ }_{r}^{t} R^{(0)}{ }_{s}^{u}{ }_{t}^{v} R_{u \bar{m} v \bar{r}}^{(0)}\right] *{ }^{(0)} 1
\end{aligned}
$$

Where we see directly that in the reduction $\mathcal{Z}_{3}=\mathcal{Z}_{4}=\mathcal{Z}_{5}=\mathcal{Z}_{6}=0$. The result above represents the only terms in the reduction result that can not be expressed in terms of $Z_{m \bar{m} n \bar{n}}$ for arbitrary choice of the parameters $a_{1}$ and $a_{2}$. For this reason we now make the choice $a_{1}=a_{2}$ which then allows the result to be rewritten as

$$
\left.\int \hat{s}_{18}(\hat{\nabla} \hat{G})^{2} \hat{R}^{2} \hat{*} 1\right|_{\text {kin }}=\frac{192\left(1+a_{1}\right)}{2 \kappa_{11}^{2}} \int_{\mathcal{M}_{3}} F^{i} \wedge * F^{j} \int_{Y_{4}}\left(i Z_{m \bar{n}} \omega_{i}^{(0) \bar{r} m} \omega_{j}^{(0) \bar{n}} \overline{\bar{r}}+2 Z_{m \bar{n} r \bar{s}} \omega_{i}^{(0) \bar{n} m} \omega_{j}^{(0) \bar{s} r}\right) *^{(0)} 1 .
$$

Furthermore we note that if the basis (A.4) is reduced with and arbitrary set of coefficients and then we demand that the result can be expressed in terms of $Z_{m \bar{m} n \bar{n}}$, then only a multiple of the linear combination

$$
\int_{\mathcal{M}_{3}} F^{i} \wedge * F^{j} \int_{Y_{4}}\left(i Z_{m \bar{n}} \omega_{i}^{(0) \bar{r} m} \omega_{j}^{(0) \bar{n}} \bar{r}+2 Z_{m \bar{n} r \bar{s}} \omega_{i}^{(0) \bar{n} m} \omega_{j}^{(0) \bar{s} r}\right) *^{(0)} 1
$$

is produced.

Open Access. This article is distributed under the terms of the Creative Commons Attribution License (CC-BY 4.0), which permits any use, distribution and reproduction in any medium, provided the original author(s) and source are credited. 


\section{References}

[1] C. Vafa, Evidence for F-theory, Nucl. Phys. B 469 (1996) 403 [hep-th/9602022] [INSPIRE].

[2] K. Becker and M. Becker, M theory on eight manifolds, Nucl. Phys. B 477 (1996) 155 [hep-th/9605053] [INSPIRE].

[3] K. Becker and M. Becker, Supersymmetry breaking, M-theory and fluxes, JHEP 07 (2001) 038 [hep-th/0107044] [INSPIRE].

[4] T.W. Grimm, T.G. Pugh and M. Weissenbacher, On M-theory fourfold vacua with higher curvature terms, Phys. Lett. B 743 (2015) 284 [arXiv:1408.5136] [INSPIRE].

[5] H. Lü, C.N. Pope, K.S. Stelle and P.K. Townsend, Supersymmetric deformations of $G_{2}$ manifolds from higher order corrections to string and M-theory, JHEP 10 (2004) 019 [hep-th/0312002] [INSPIRE].

[6] H. Lü, C.N. Pope, K.S. Stelle and P.K. Townsend, String and M-theory deformations of manifolds with special holonomy, JHEP 07 (2005) 075 [hep-th/0410176] [INSPIRE].

[7] D. Prins and D. Tsimpis, Type IIA supergravity and M-theory on manifolds with SU(4) structure, Phys. Rev. D 89 (2014) 064030 [arXiv:1312.1692] [INSPIRE].

[8] D. Nemeschansky and A. Sen, Conformal invariance of supersymmetric $\sigma$ models on Calabi-Yau manifolds, Phys. Lett. B 178 (1986) 365 [InSPIRE].

[9] K. Becker, D. Robbins and E. Witten, The $\alpha^{\prime}$ expansion on a compact manifold of exceptional holonomy, JHEP 06 (2014) 051 [arXiv:1404.2460] [INSPIRE].

[10] E. Cremmer, B. Julia and J. Scherk, Supergravity theory in eleven-dimensions, Phys. Lett. B 76 (1978) 409 [INSPIRE].

[11] M.J. Duff, J.T. Liu and R. Minasian, Eleven-dimensional origin of string-string duality: a one loop test, Nucl. Phys. B 452 (1995) 261 [hep-th/9506126] [InSPIRE].

[12] M.B. Green and P. Vanhove, D instantons, strings and M-theory, Phys. Lett. B 408 (1997) 122 [hep-th/9704145] [INSPIRE].

[13] M.B. Green, M. Gutperle and P. Vanhove, One loop in eleven-dimensions, Phys. Lett. B 409 (1997) 177 [hep-th/9706175] [INSPIRE].

[14] E. Kiritsis and B. Pioline, On $R^{4}$ threshold corrections in IIB string theory and $(p, q)$ string instantons, Nucl. Phys. B 508 (1997) 509 [hep-th/9707018] [INSPIRE].

[15] J.G. Russo and A.A. Tseytlin, One loop four graviton amplitude in eleven-dimensional supergravity, Nucl. Phys. B 508 (1997) 245 [hep-th/9707134] [INSPIRE].

[16] I. Antoniadis, S. Ferrara, R. Minasian and K.S. Narain, $R^{4}$ couplings in $M$ and type-II theories on Calabi-Yau spaces, Nucl. Phys. B 507 (1997) 571 [hep-th/9707013] [INSPIRE].

[17] A.A. Tseytlin, $R^{4}$ terms in 11 dimensions and conformal anomaly of $(2,0)$ theory, Nucl. Phys. B 584 (2000) 233 [hep-th/0005072] [InSPIRE].

[18] J.T. Liu and R. Minasian, Higher-derivative couplings in string theory: dualities and the B-field, Nucl. Phys. B 874 (2013) 413 [arXiv: 1304.3137] [InSPIRE].

[19] K. Peeters, J. Plefka and S. Stern, Higher-derivative gauge field terms in the M-theory action, JHEP 08 (2005) 095 [hep-th/0507178] [INSPIRE].

[20] T.W. Grimm, T.G. Pugh and M. Weissenbacher, The effective action of warped M-theory reductions with higher-derivative terms - part II, JHEP 12 (2015) 117 [arXiv:1507.00343] [INSPIRE]. 
[21] S. Katmadas and R. Minasian, $N=2$ higher-derivative couplings from strings, JHEP 02 (2014) 093 [arXiv: 1311.4797] [INSPIRE].

[22] K. Dasgupta, G. Rajesh and S. Sethi, $M$ theory, orientifolds and G-flux, JHEP 08 (1999) 023 [hep-th/9908088] [INSPIRE].

[23] S.B. Giddings, S. Kachru and J. Polchinski, Hierarchies from fluxes in string compactifications, Phys. Rev. D 66 (2002) 106006 [hep-th/0105097] [INSPIRE].

[24] S.B. Giddings and A. Maharana, Dynamics of warped compactifications and the shape of the warped landscape, Phys. Rev. D 73 (2006) 126003 [hep-th/0507158] [INSPIRE].

[25] C.P. Burgess et al., Warped supersymmetry breaking, JHEP 04 (2008) 053 [hep-th/0610255] [INSPIRE].

[26] G. Shiu, G. Torroba, B. Underwood and M.R. Douglas, Dynamics of warped flux compactifications, JHEP 06 (2008) 024 [arXiv:0803.3068] [INSPIRE].

[27] M.R. Douglas and G. Torroba, Kinetic terms in warped compactifications, JHEP 05 (2009) 013 [arXiv:0805.3700] [INSPIRE].

[28] L. Martucci, On moduli and effective theory of $N=1$ warped flux compactifications, JHEP 05 (2009) 027 [arXiv: 0902.4031] [INSPIRE].

[29] B. Underwood, A breathing mode for warped compactifications, Class. Quant. Grav. 28 (2011) 195013 [arXiv:1009.4200] [INSPIRE].

[30] A.R. Frey and J. Roberts, The dimensional reduction and Kähler metric of forms in flux and warping, JHEP 10 (2013) 021 [arXiv:1308.0323] [INSPIRE].

[31] L. Martucci, Warping the Kähler potential of F-theory/IIB flux compactifications, JHEP 03 (2015) 067 [arXiv:1411.2623] [INSPIRE].

[32] T.W. Grimm, D. Klevers and M. Poretschkin, Fluxes and warping for gauge couplings in F-theory, JHEP 01 (2013) 023 [arXiv: 1202.0285] [INSPIRE].

[33] T.W. Grimm, R. Savelli and M. Weissenbacher, On $\alpha^{\prime}$ corrections in $N=1$ F-theory compactifications, Phys. Lett. B $\mathbf{7 2 5}$ (2013) 431 [arXiv: 1303.3317] [INSPIRE].

[34] T.W. Grimm, J. Keitel, R. Savelli and M. Weissenbacher, From M-theory higher curvature terms to $\alpha^{\prime}$ corrections in F-theory, Nucl. Phys. B 903 (2016) 325 [arXiv:1312.1376] [INSPIRE].

[35] D. Junghans and G. Shiu, Brane curvature corrections to the $N=1$ type-II/F-theory effective action, JHEP 03 (2015) 107 [arXiv: 1407.0019] [INSPIRE].

[36] M. Haack and J. Louis, Duality in heterotic vacua with four supercharges, Nucl. Phys. B 575 (2000) 107 [hep-th/9912181] [INSPIRE].

[37] M. Haack and J. Louis, M theory compactified on Calabi-Yau fourfolds with background flux, Phys. Lett. B 507 (2001) 296 [hep-th/0103068] [INSPIRE].

[38] T.W. Grimm and H. Hayashi, F-theory fluxes, chirality and Chern-Simons theories, JHEP 03 (2012) 027 [arXiv: 1111.1232] [INSPIRE].

[39] M. Cvetič, T.W. Grimm and D. Klevers, Anomaly cancellation and Abelian gauge symmetries in F-theory, JHEP 02 (2013) 101 [arXiv:1210.6034] [INSPIRE].

[40] J. Gray and A. Lukas, Gauge five-brane moduli in four-dimensional heterotic models, Phys. Rev. D 70 (2004) 086003 [hep-th/0309096] [INSPIRE]. 